\title{
Not proper ROC curves as new tool for the analysis of differentially expressed genes in microarray experiments Stefano Parodi*1, Vito Pistoia ${ }^{2}$ and Marco Muselli ${ }^{3}$
}

\author{
Address: ${ }^{1}$ Epidemiology and Biostatistics Section, Scientific Directorate, G. Gaslini Children's Hospital, Genoa, Italy, ${ }^{2}$ Laboratory of Oncology, G. \\ Gaslini Children's Hospital, Genoa, Italy and ${ }^{3}$ Institute of Electronics, Computer and Telecommunication Engineering, Italian National Research \\ Council, Genoa, Italy \\ Email: Stefano Parodi* - stefanoparodi@ospedale-gaslini.ge.it; Vito Pistoia - vitopistoia@ospedale-gaslini.ge.it; \\ Marco Muselli - marco.muselli@ieiit.cnr.it \\ * Corresponding author \\ Published: 3 October 2008 \\ BMC Bioinformatics 2008, 9:410 doi:10.1186/147|-2105-9-410 \\ Received: 23 May 2008 \\ Accepted: 3 October 2008 \\ This article is available from: http://www.biomedcentral.com//47I-2105/9/410 \\ (C) 2008 Parodi et al; licensee BioMed Central Ltd. \\ This is an Open Access article distributed under the terms of the Creative Commons Attribution License (http://creativecommons.org/licenses/by/2.0), \\ which permits unrestricted use, distribution, and reproduction in any medium, provided the original work is properly cited.
}

\begin{abstract}
Background: Most microarray experiments are carried out with the purpose of identifying genes whose expression varies in relation with specific conditions or in response to environmental stimuli. In such studies, genes showing similar mean expression values between two or more groups are considered as not differentially expressed, even if hidden subclasses with different expression values may exist. In this paper we propose a new method for identifying differentially expressed genes, based on the area between the ROC curve and the rising diagonal $(A B C R) . A B C R$ represents a more general approach than the standard area under the ROC curve (AUC), because it can identify both proper (i.e., concave) and not proper ROC curves (NPRC). In particular, NPRC may correspond to those genes that tend to escape standard selection methods.

Results: We assessed the performance of our method using data from a publicly available database of 4026 genes, including 14 normal B cell samples (NBC) and 20 heterogeneous lymphomas (namely: 9 follicular lymphomas and II chronic lymphocytic leukemias). Moreover, NBC also included two sub-classes, i.e., 6 heavily stimulated and 8 slightly or not stimulated samples. We identified 1607 differentially expressed genes with an estimated False Discovery Rate of $15 \%$. Among them, 16 corresponded to NPRC and all escaped standard selection procedures based on $A \cup C$ and $t$ statistics. Moreover, a simple inspection to the shape of such plots allowed to identify the two subclasses in either one class in 13 cases (81\%).
\end{abstract}

Conclusion: NPRC represent a new useful tool for the analysis of microarray data.

\section{Background}

Microarray technology allows to analyze the expression of thousands of genes in a single experiment [1]. The identification of genes whose expression changes in pathological conditions or upon exposure to stimuli, such as pharmacologic treatment, is a very common aim of microarray-based studies. In this respect, different statistical tests, generally based on measures of distance between classes, have been so far proposed [2-4]. Among them, two parameters of Receiver Operating Characteristic (ROC) curves, namely the area under the curve (AUC), and the partial area at a selected high specificity threshold ( $p A U C)$, have been applied for such a purpose [4-8]. A ROC curve represents the relationship between the true 
positive fraction (TPF) and the false positive fraction (FPF) resulting from a set of binary classification tests based on each possible decision threshold value $[5,9]$. TPF is commonly known as Sensitivity, while FPF corresponds to $1-$ Specificity. When a ROC curve is drawn using a specific gene expression profile, AUC estimates the probability that a subject randomly selected from one class (e.g., a group of individuals affected by a specific disease) has an expression value higher than a subject randomly selected from the other class (e.g., healthy individuals) [6].

In case of unimodal distributions in the two classes with similar variance and different mean, the corresponding ROC curve tends to lie completely above the diagonal line and to be concave ("proper" ROC curve, e.g., Curve I in Figure 1). In the case of two unimodal distributions with similar mean and variance in the two classes, the corresponding ROC curve will approach the rising diagonal (Curve II in Figure 1). This particular ROC curve is often named the "chance line", because it represents the set of all the possible statistical tests with equal probability for a true positive and a false positive result, i.e., corresponding to the set of results expected by chance alone. In such a case $A U C$ will tend to 0.5 . However, $A U C$ values close to 0.5 may also be obtained from genes differentially expressed among two classes, when the presence of a hidden bimodal or multimodal distribution in either class causes the ROC curve to cross the chance line [10], like the not concave (not proper) Curve III and Curve IV in Figure 1. Bimodal or multi-modal distributions within a class may indicate the presence of unknown subclasses with different expression values [10]. As a consequence, the identification of such subclasses may provide useful insights about biological mechanisms underlying physiologic or pathologic conditions. However, most expression profiles corresponding to not proper ROC curves (NPRC) are likely to be discharged by the commonly used feature selection methods (including AUC, pAUC and Student's $t$ statistics), because either mean or median values tend to be similar between the considered groups.

To allow the identification of different kind of differentially expressed genes, we have developed a new statistical method of feature selection based on the area between the ROC curve and the rising diagonal $(A B C R)$. Furthermore, to separate NPRC (like Curve III and Curve IV in Figure 1) from both uninformative and proper ROC plots (like Curve II and Curve I, respectively) we have developed a new approach based on the combination of standard feature selection procedures based either on AUC or on $t$ test with a new statistical test based on a simple variant of $A B C R$ (TNRC $=$ Test for Not-proper ROC Curves).

The performance of our method was evaluated by comparing the gene expression profiles in two different classes, using data from a publicly available data base including 4026 gene expression profiles [11]. Class A included 14 different samples of normal circulating B cells (NBC), class B included 20 heterogeneous lymphomas. Class A and B both included two subclasses, namely: 6 heavily stimulated and 8 slightly stimulated or unstimulated samples in class A (Table 1); 9 follicular lymphomas (FL) and 11 chronic lymphocytic leukemias (CLL) in class B.

The aim of this study is to illustrate a new comprehensive approach based on the combination of both standard (AUC) and new (ABCR and TNRC) ROC parameters. Moreover, we show how not proper ROC curves, identified by TNRC, may allow at the same time both to select differentially expressed genes that tend to escape standard statistical tools, and to point out the presence of hidden subclasses with biological or clinical meaning. For such purposes, we selected the genes with the highest $A B C R$ value corresponding to an a priori chosen False Discovery Rate (FDR) [12]. Among the expression profiles selected by $A B C R$ we identified over-expressed and underexpressed genes using either the Area Under the ROC curve (AUC) or the Student's $t$ statistic, which both represent standard methods for feature selection in microarray analysis $[3,4,8]$. NPRC were identified by high values of TNRC statistic. A conventional unadjusted $\mathrm{p}$ value of 0.05 was used as threshold in each analysis. Furthermore, we conducted a detailed analysis of each selected NPRC, to assess the concordance between the observed gene expression and the presence of hidden subclasses (see Material and Methods for more details).

The FDR of both standard (AUC and $t$ value) and new proposed statistics (ABCR and TNRC) was also estimated under some different distribution hypotheses and at different sample size by using artificial data sets containing 4000 simulated gene expression profiles in two classes. Finally, the distribution of $A B C R$ and TNRC at some different sample size under the null hypothesis of no differentially expressed genes between two classes was estimated by extensive random permutation analysis.

\section{Results}

We grouped all the genes discussed below as follows: lymphocyte/macrophage related genes (group 1), major histocompatibility complex related genes (group 2), genes involved in malignant cell transformation (group 3), genes related to nucleic acid metabolism or DNA transcription (group 4), and gene encoding various enzymes/ kinases and other proteins (group 5). In spite of some overlap, this classification allows to subdivide the tested genes according to their functional features. 


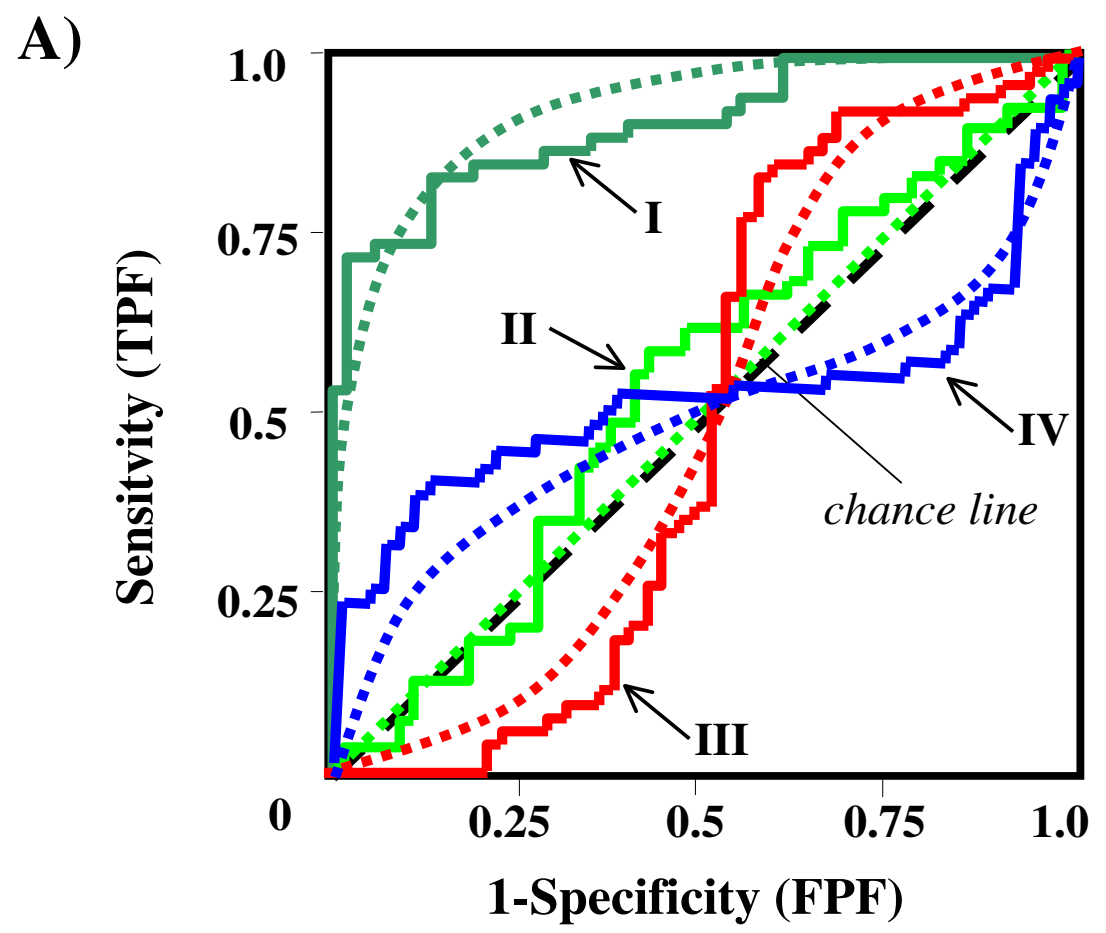

B)
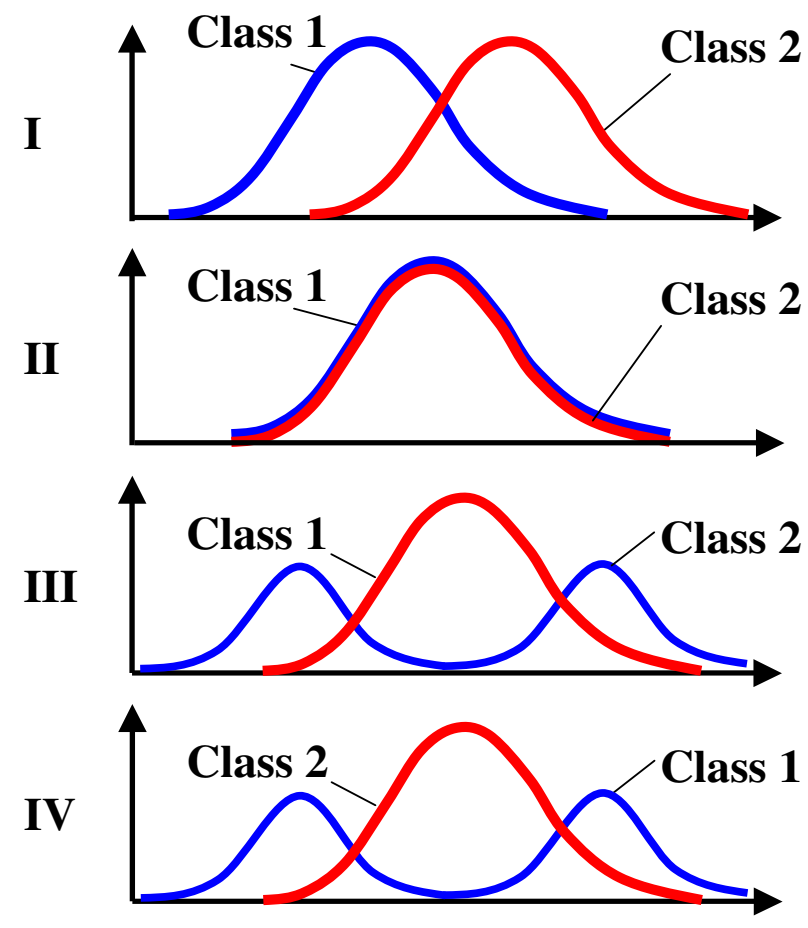

Gene expression values (arbitrary units)

Figure I

Theoretical (dotted lines) and empirical (solid lines) ROC curves (panel A) and the corresponding distribution of gene expression values (panel B). Empirical ROC curves were obtained using 50 samples randomly selected from each class. 
Table I: Pattern of stimulation of the 14 normal circulating B cells in class $A$

\begin{tabular}{ll}
\hline N & Pattern of stimulation \\
\hline & Heavily stimulated cells \\
I & Blood B cells;anti-IgM+CD40L low $48 \mathrm{~h}$ \\
2 & Blood B cells;anti-IgM+CD40L high $48 \mathrm{~h}$ \\
3 & Blood B cells;anti-IgM+CD40L 24 h \\
4 & Blood B cells;anti-IgM 24 h \\
5 & Blood B cells;anti-IgM+IL-4 24 h \\
6 & Blood B cells;anti-IgM+CD40L+IL-4 24 h \\
& Slightly or not stimulated cells \\
7 & Blood B cells;anti-IgM+IL-4 6 h \\
8 & Blood B cells;anti-IgM 6 h \\
9 & Blood B cells;anti-IgM+CD40L 6 h \\
I0 & Blood B cells;anti-IgM+CD40L+IL-4 6 h \\
II & Blood B cells;memory CD27+ \\
I2 & Blood B cells;naive CD27- \\
I3 & Blood B cells \\
I4 & Cord Blood B
\end{tabular}

Note: sample number $(\mathrm{N})$ corresponds to the original location of each sample in the original data set: http://lmpp.nih.gov/lymphoma/ data/figurel/figurel.cdt.

Our method identified 1607 genes with the highest value of $A B C R$ at a selected FDR of $15 \%$. The estimated selection probability ranged from $40 \%$ to $100 \%$ (median value: $79.5 \%$ ). Figure $2 \mathrm{~A}$ shows the results of the selection procedure combining the new proposed TNRC statistic with the standard AUC on the subset of 1607 genes selected by $A B C R$. Area I includes 16 genes with a statistically significant TNRC value (blue circles), while areas II and III collect 1524 genes with a statistically significant $A U C$ value. In particular, genes in area II (green circles) were found to be under-expressed in class A compared with class $\mathrm{B}$, while genes in area III (red circles) were found to be overexpressed in class A. No genes corresponding to NPRC were identified by AUC statistic and vice versa. However, 67 genes (empty circles in Figure 2A) were not identified by either $A U C$ or TNRC. The large majority of them $(\mathrm{n}=$ 57) had a borderline statistically significant value of $A U C$ ( $\mathrm{p}$ value between 0.05 and 0.10 ), but not for TNRC, while 2 genes had a borderline $\mathrm{p}$ value for TNRC, but not for $A U C$. The analysis was repeated using $t$ statistic in place of $A U C$, and the corresponding results are summarized in Figure 2B. Also in this case, no gene with statistically significant TNRC value was identified by the standard statistical method (i.e., $t$ value). However, the separation between this two different kind of differentially expressed genes was less evident than that obtained by using AUC (Figure 2A). Moreover, a larger number of genes $(\mathrm{n}=112)$ remained unclassified (empty circles), including some genes with a very low value for both TNRC and $t$ statistics.

Table 2 shows the 16 genes with the highest TNRC value, corresponding to the blue circles in Figure $2 \mathrm{~A}$ and $2 \mathrm{~B}$.
Among them, 4 had an unknown function. The others belonged to group 1 (genes n. 1-3, and n.13), group 3 (genes n. 4 and, 8), group 4 (gene n. 5), or group 5 (genes n. $6,7,11,12$, and 15) [13]. Values of TNRC parameter ranged from 0.082 to 0.2 , while the corresponding $A B C R$ values ranged between 0.202 and 0.253 . Interestingly, the first three selected genes in Table 2 included all the clones of the gene for Immunoglobulin $\mathrm{J}$ chain in the original data set. Furthermore, the gene n. 6 (VRK2 kinase) was present in another clone in the same data set (gene n. 12).

Table 3 shows the 16 top genes selected by the standard ROC analysis based on AUC values among the 1607 genes reported in Figure 2A. Genes were sorted on the basis of the corresponding pure accuracy (i.e., the probability to correctly rank two samples, one randomly extracted from class A and one from class B), which is estimated by AUC for proper curves lying above the chance line, and by 1 AUC for proper curves lying below. Genes in Table 3 also corresponded to the 16 highest values of $A B C R$. Eleven genes showed an $A U C<0.5$ and they were accordingly considered as under-expressed in FL, CLL in comparison with NBC, while the remaining 5 genes showed an AUC > 0.5 and were considered as over-expressed. Among the under-expressed genes two belonged to group 1 (genes $\mathrm{n}$. 9 and n. 15), 1 to group 3 (gene n.5), 4 to group 4 (genes n. 2, n. 4 , n. 6 and n.14), 3 to group 5 (Genes n.3, n. 8 and n. 16), and 1 had an unknown function (gene n. 13). Among the over-expressed genes, 1 belonged to group 1 (gene n. 7), 1 to group 4 (gene n. 12), and 3 had unknown functions (genes n. 1, n. 10, and n. 11) [13]. AUC ranged between 0 and 0.02 in under-expressed genes, and between 0.986 and 1 in over-expressed genes. $A B C R$ values ranged between 0.479 and 0.5 .

All the analyses were repeated varying the FDR threshold. At $\mathrm{FDR}=10 \%, 1454$ genes were identified by $A B C R$. Among them, 1439 (99\%) were called over- or underexpressed on the basis of AUC statistic, 4 genes corresponding to NPRC were identified by TNRC and 11 were not identified by either statistic. Among these latter, 8 had a borderline statistical significant value for $A U C$ and 1 for $T N R C$. Also in this case, no genes identified by AUC were also selected by TNRC and vice versa. Finally, using FDR = $20 \%$ for $A B C R, 1866$ genes were selected. Among them, 1524 (82\%) were selected by AUC, 24 by TNRC, and 318 remained not classified, including 272 genes with a borderline statistical significance for AUC and 3 for TNRC. Also in this case no genes were identified as differentially expressed by both AUC and TNRC.

Because the main sources of heterogeneity were known $a$ priori for both class A (NBC), which included differently stimulated cells, and for class B, which included samples from two different malignant diseases (namely, FL and 


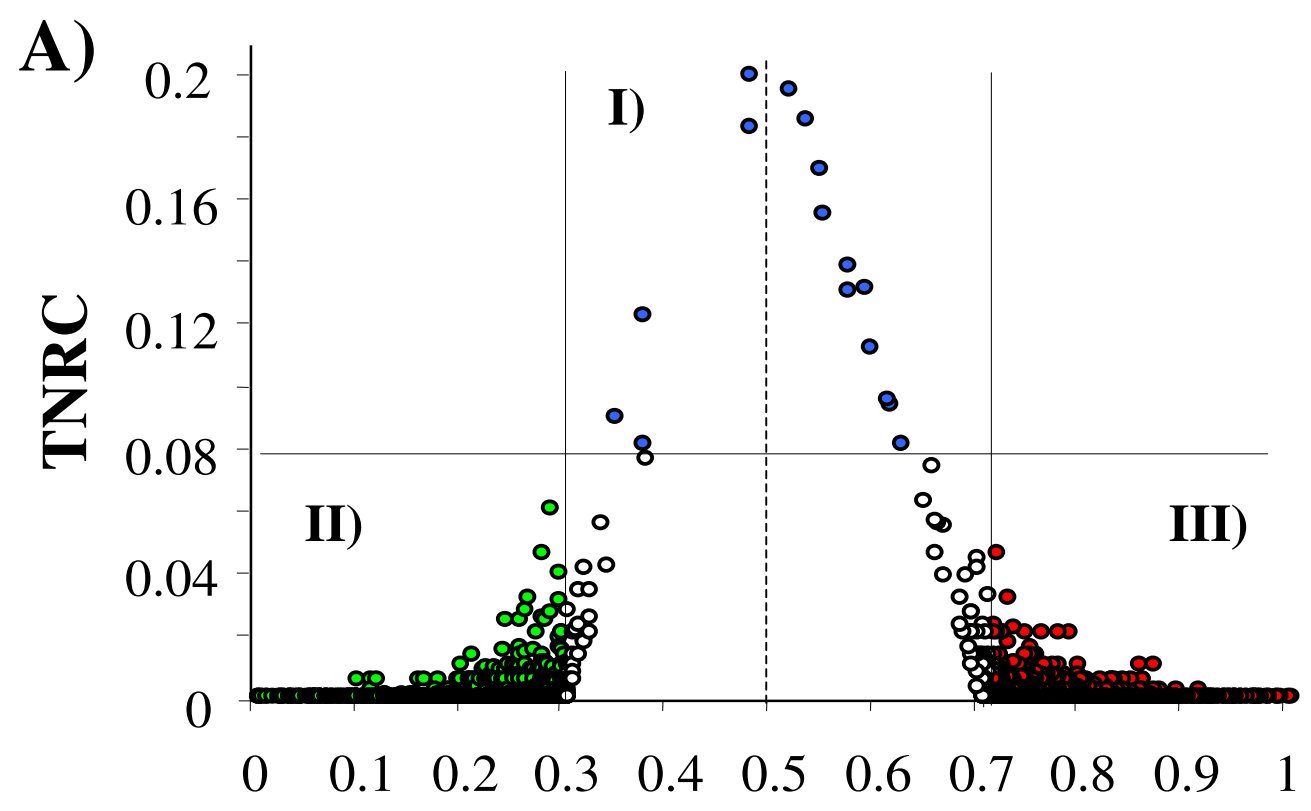

B)

\section{AUC}

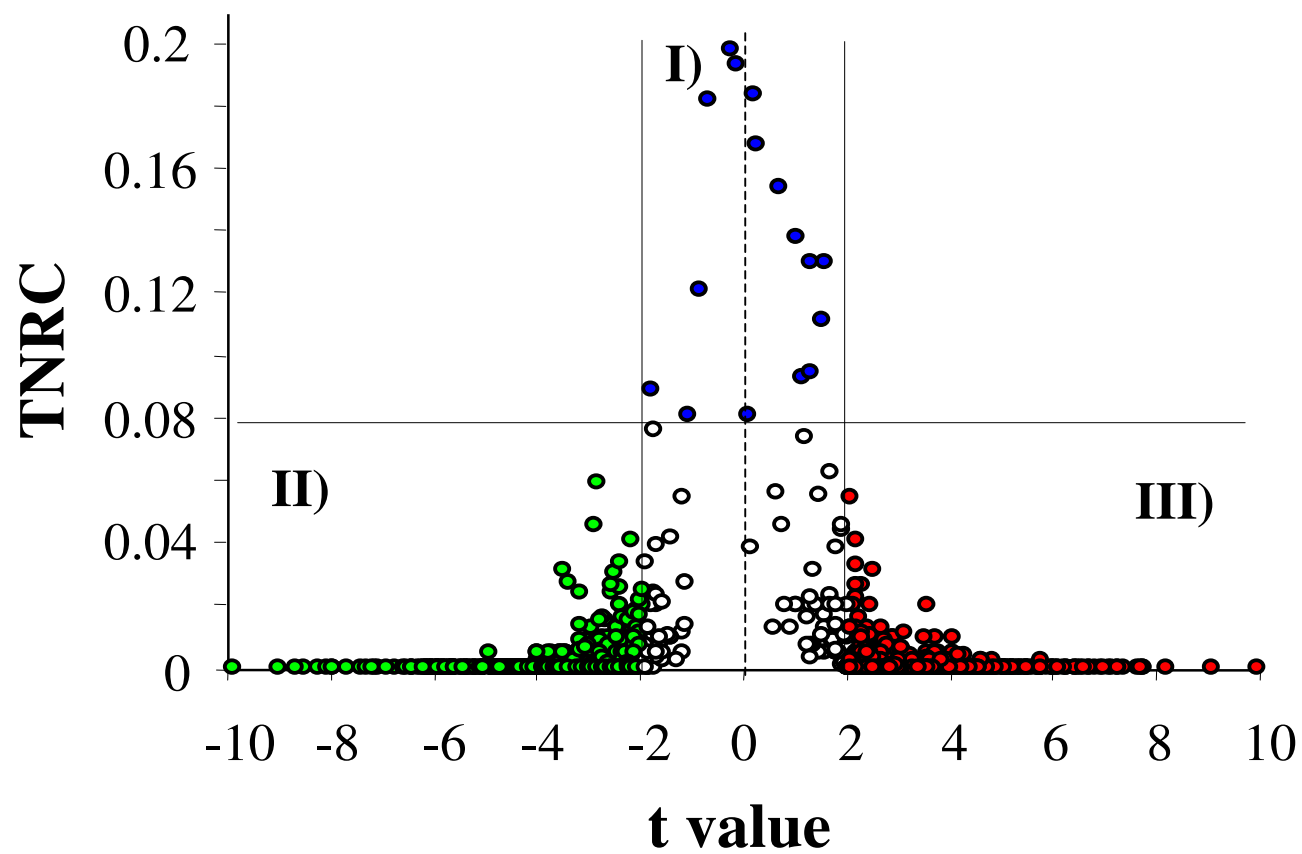

Figure 2

TNRC value for the 1607 top genes selected by $A B C R$ at FDR $=15 \%$, as a function of $A U C$ (Panel $A$ ) and $t$ statistics (Panel B). Area I includes genes corresponding to not proper ROC curves (blue circles); Area II includes genes underexpressed in malignant cells (green circles); Area III includes genes over-expressed in malignant cells (red circles); empty circles correspond to unselected genes. Solid lines represent the thresholds corresponding to $p=0.05$ for TNRC (horizontal line in Panel A and in Panel B), for AUC (vertical lines in Panel A) and for $t$ statistic (vertical lines in Panel B). Broken lines represent the expected value under the null hypothesis for AUC (Panel A) and for $t$ statistics (Panel B). 
Table 2: Comparison between I4 NBC and 20 heterogeneous lymphomas - genes selected by ABCR and TNRC statistics

\begin{tabular}{|c|c|c|c|c|c|}
\hline$N$ & Gene ID & Gene name & $A B C R$ & $T N R C$ & Group \\
\hline I & GENE3389X & Immunoglobulin J chain & 0.2250 & 0.2000 & I \\
\hline 2 & GENE3390X & Immunoglobulin J chain & 0.2096 & 0.1954 & I \\
\hline 3 & GENE3388X & Immunoglobulin J chain & 0.2143 & 0.1858 & I \\
\hline 4 & GENE3323X & $B C L 7 A$ & 0.2069 & 0.1836 & 3 \\
\hline 5 & GENE3407X & Histone deacetylase 3 & 0.2122 & 0.1694 & 4 \\
\hline 6 & GENE75X & VRK2 kinase & 0.2015 & 0.1552 & 5 \\
\hline 7 & GENEII4IX & MAPKKK5 & 0.2105 & 0.1390 & 5 \\
\hline 8 & GENEI8I7X & $B L 34$ & 0.2171 & 0.1314 & 3 \\
\hline 9 & GENE2395X & Unknown & 0.2025 & 0.1310 & Unknown \\
\hline 10 & GENE2696X & Unknown & 0.2531 & 0.1224 & Unknown \\
\hline 11 & GENE352IX & Similar to KIAA0050 & 0.2051 & 0.1122 & 5 \\
\hline 12 & GENE74X & VRK2 kinase & 0.2043 & 0.0954 & 5 \\
\hline 13 & GENE2287X & MRC OX-2 & 0.2046 & 0.0940 & I \\
\hline 14 & GENE354IX & Unknown & 0.2436 & 0.0900 & Unknown \\
\hline 15 & GENEI362X & Syndecan-2 & 0.2031 & 0.0816 & 5 \\
\hline 16 & GENE2673X & Unknown & 0.2034 & 0.0816 & Unknown \\
\hline
\end{tabular}

Group I: lymphocyte/macrophage related genes; Group 2: major histocompatibility complex related genes; Group 3: genes involved in malignant cell transformation; Group 4: genes related to nucleic acid metabolism or DNA transcription; Group 5: genes encoding various enzymes/kinases and other proteins. ABCR: Area between the ROC curve and the rising diagonal; TNRC: Test for not-proper ROC curves.

CLL), we carried out a detailed analysis of each ROC curve obtained from the expression values of genes listed in Table 2 (Figures 3, 4, 5, 6, 7, 8, 9, 10, 11, 12, 13, 14, 15, 16,17 and 18). Figures from 3 to 18 were ordered according to the ranks of the corresponding genes in Table 2, i.e., Figure 3 refers to the expression of gene n. 1, Figure 4 corresponds to gene n. 2, and so on. Each plot reports both the origin of samples in class B (i.e., either FL or CLL) and the two major subclasses within NBC class, according to Table 1 (i.e., heavily stimulated and slightly or not stimulated cells). Moreover, each plot was arbitrarily split into two parts to roughly separate samples with high (left side) and with low (right side) expression level. Finally, the ROC curves in Figures 3, 4, 5, 6, 7, 8, 9, 10, 11, 12, 13, 14, $15,16,17.18$ were classified as "sigmoid-shaped" (like Curve III in Figure 1) and "inversely sigmoid-shaped" (like Curve IV in Figure 1).

Six out of the 16 genes in Table 2 corresponded to sigmoid-shaped curves (Figures 7, 8, 13, 14, 17, and 18). Four curves allowed to identify the two hidden subgroups of highly stimulated and slightly or not stimulated B cells,

Table 3: Comparison between I4 NBC and 20 heterogeneous lymphomas - top 16 genes selected by ABCR and AUC statistics

\begin{tabular}{|c|c|c|c|c|c|c|}
\hline $\mathrm{N}$ & Gene ID & & Gene name & $A B C R$ & AUC & Group \\
\hline I & GENE2495X & + & Unknown & 0.5000 & 1.0000 & Unknown \\
\hline 2 & GENEI $217 X$ & - & NFkB2 & 0.5000 & 0.0000 & 4 \\
\hline 3 & GENEI $602 X$ & - & protein kinase (zpk) & 0.5000 & 0.0000 & 5 \\
\hline 4 & GENEII9IX & - & CREM & 0.5000 & 0.0000 & 4 \\
\hline 5 & GENEII7IX & - & Similar to spi-I & 0.4964 & 0.0036 & 3 \\
\hline 6 & GENEI219X & - & IkB alpha & 0.4964 & 0.0036 & 4 \\
\hline 7 & GENE3795X & + & AIM2 & 0.4964 & 0.9964 & I \\
\hline 8 & GENEI730X & - & Sgk & 0.4929 & 0.0071 & 5 \\
\hline 9 & GENEII70X & - & CD83 & 0.4929 & 0.0071 & I \\
\hline 10 & GENE3702X & + & Unknown & 0.4893 & 0.9893 & Unknown \\
\hline 11 & GENE2494X & + & Unknown & 0.4857 & 0.9857 & Unknown \\
\hline 12 & GENE3280X & + & elF-2B alpha subunit & 0.4857 & 0.9857 & 4 \\
\hline 13 & GENEII60X & - & Unknown & 0.4857 & 0.0143 & Unknown \\
\hline 14 & GENE589X & - & elF-3 & 0.4821 & 0.0179 & 4 \\
\hline 15 & GENEII $72 X$ & - & CD83 & 0.4786 & 0.0214 & I \\
\hline 16 & GENE324X & - & Nakl & 0.4786 & 0.0214 & 5 \\
\hline
\end{tabular}

Group I: lymphocyte/macrophage related genes; Group 2: major histocompatibility complex related genes; Group 3: genes involved in malignant cell transformation; Group 4: genes related to nucleic acid metabolism or DNA transcription; Group 5: genes encoding various enzymes/kinases and other proteins. ABCR: Area between the ROC curve and the rising diagonal; AUC = Area under the ROC curve; "+" = overexpressed; "-" = underexpressed. 


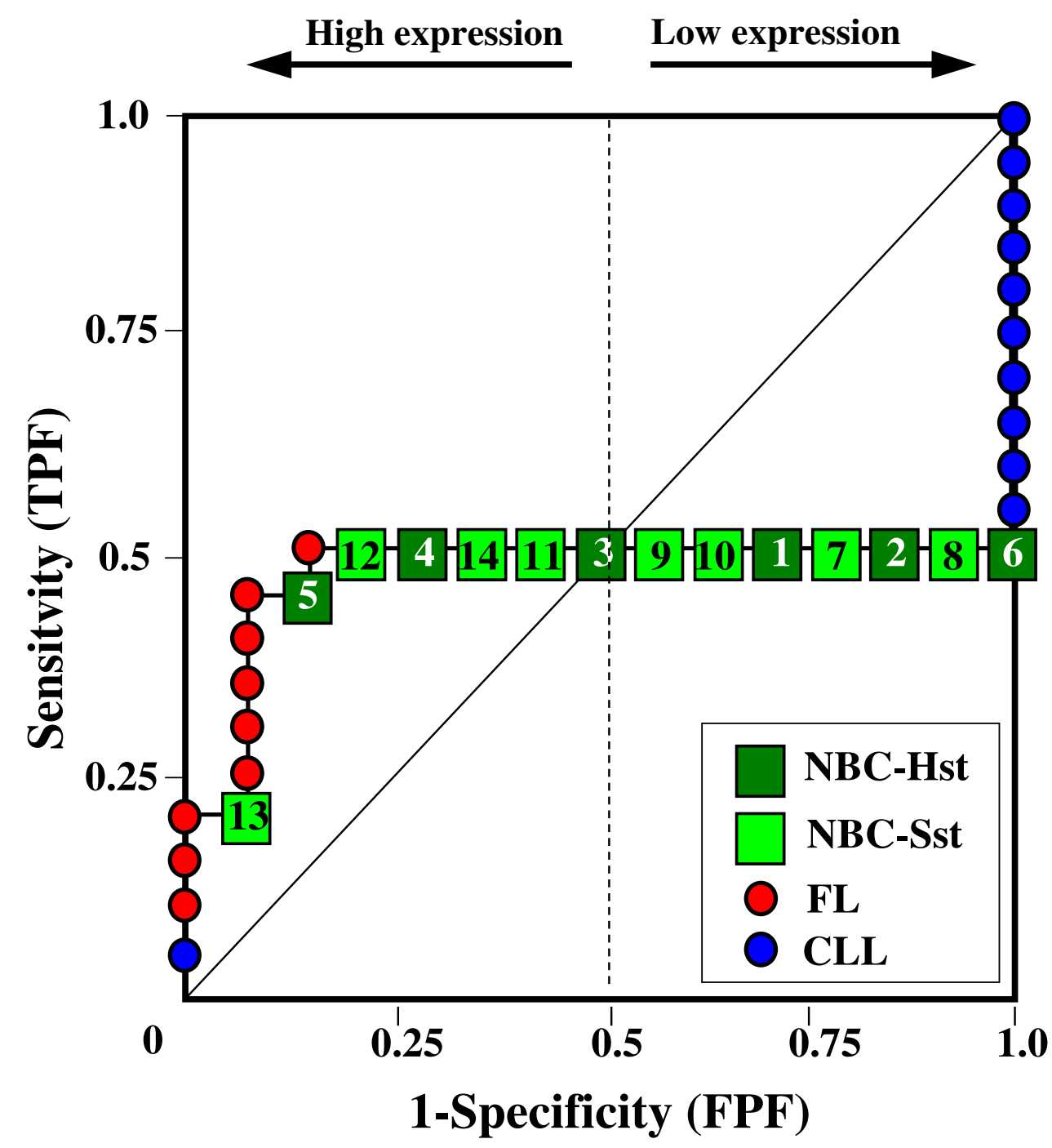

\section{FL CLL}

High expression

Low expression

\begin{tabular}{|c|c|}
\hline 9 & 1 \\
\hline 0 & 10 \\
\hline
\end{tabular}

\section{$P<0.001$}

Figure 3

Not proper ROC curve corresponding to the expression of gene $n$. I in Table 2 (GENE3389X: Immunoglobulin J Chain). Comparison between class A ( 14 samples of NBC) and class B (20 heterogeneous lymphomas, including $9 \mathrm{FL}$ and II CLL samples). Hst = Highly stimulated NBC; SSt= Slightly or not stimulated NBC (Table I). NBC samples are numbered according to Table I. 


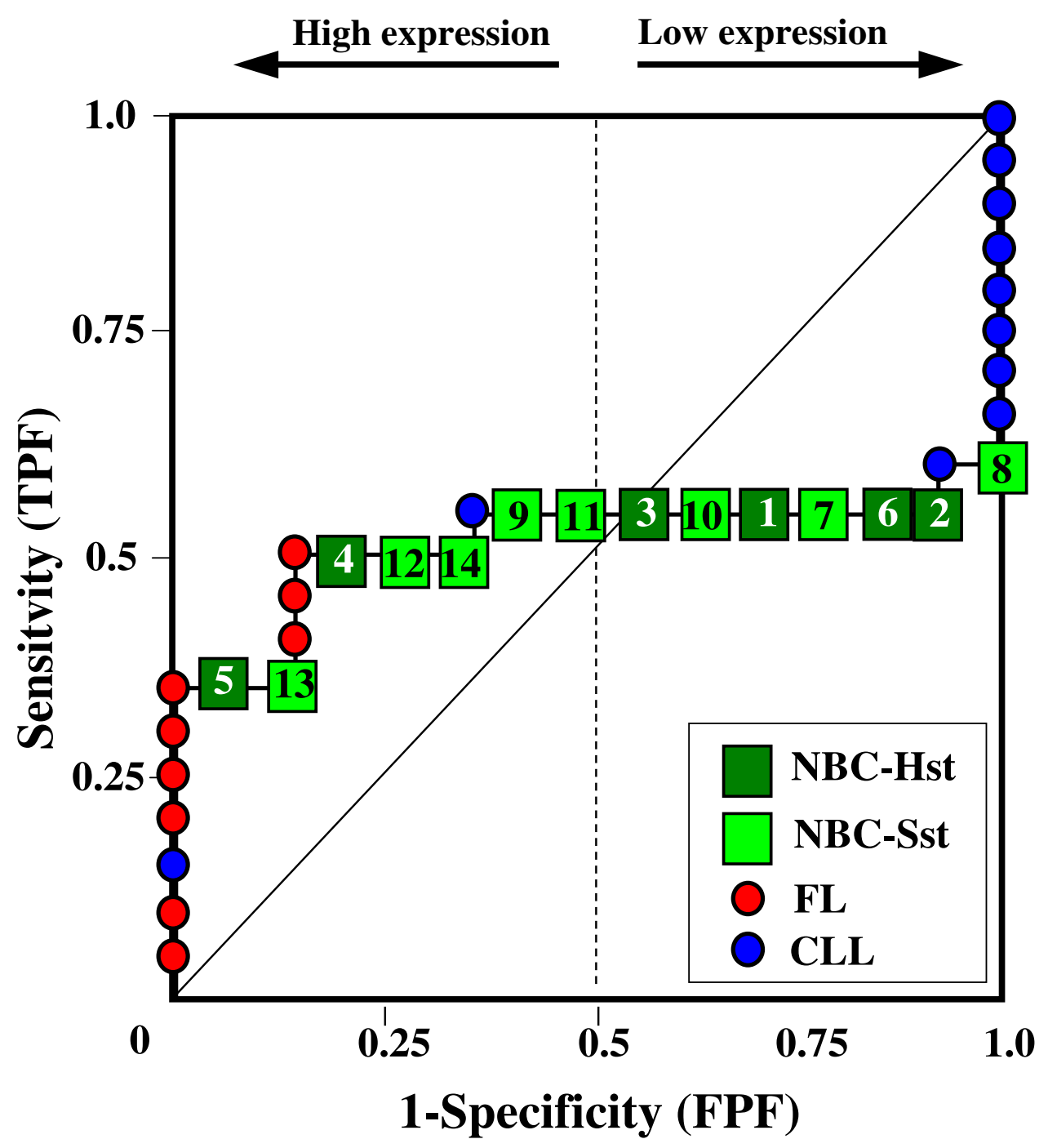

FL CLL

High expression

Low expression

\begin{tabular}{|l|l|}
\hline 9 & 2 \\
\hline 0 & 9 \\
\hline
\end{tabular}

\section{$P<0.001$}

Figure 4

Not proper ROC curve corresponding to the expression of gene $\mathbf{n} .2$ in Table 2 (GENE3390X: Immunoglobulin J Chain). Comparison between class A (I4 samples of NBC) and class B (20 heterogeneous lymphomas, including 9 FL and II CLL samples). Hst = Highly stimulated NBC; SSt= Slightly or not stimulated NBC (Table I). NBC samples are numbered according to Table I. 


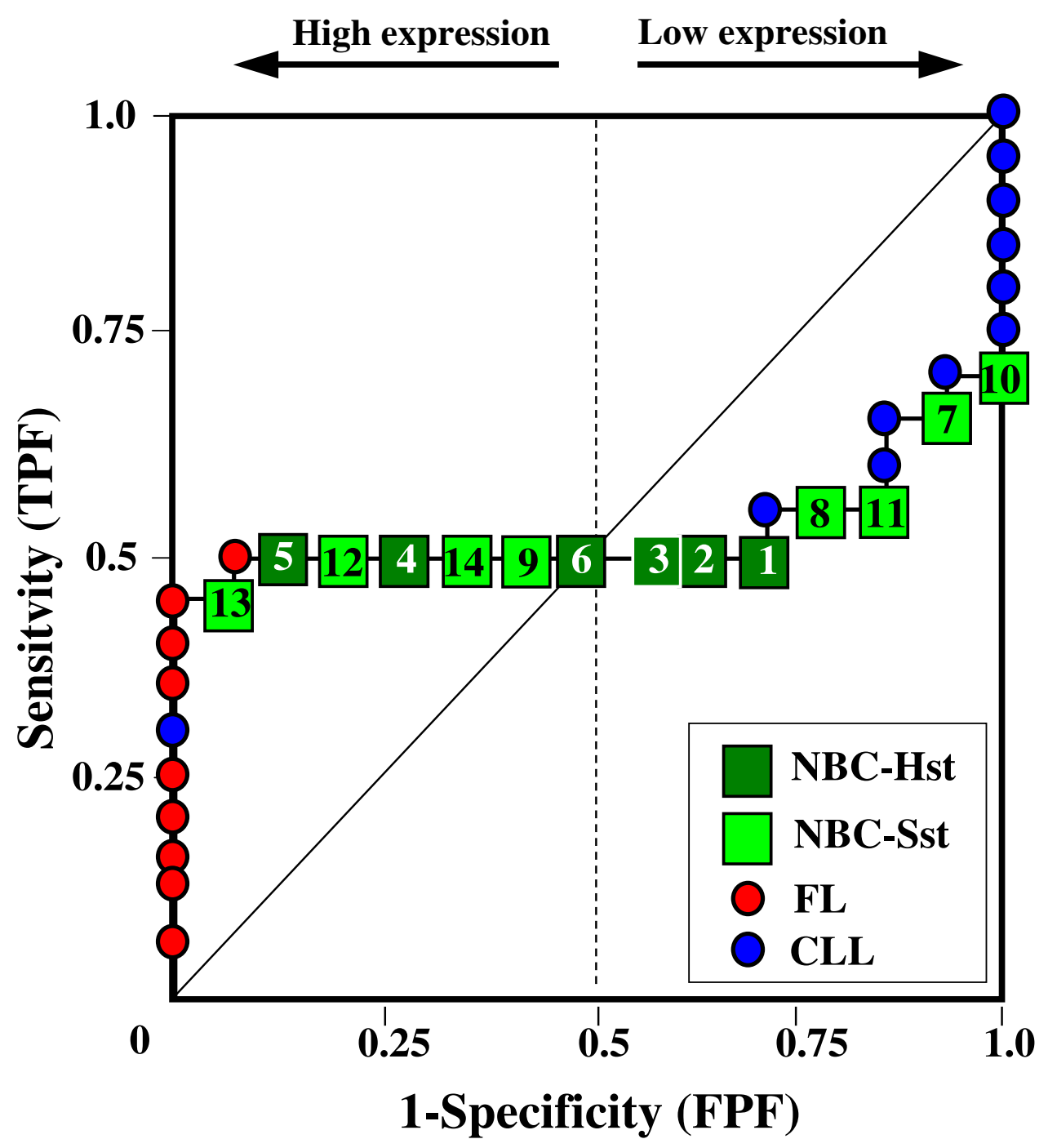

\section{FL CLL}

High expression

Low expression

\begin{tabular}{|c|c|}
\hline 9 & 1 \\
\hline 0 & 10 \\
\hline
\end{tabular}

\section{$P<0.001$}

Figure 5

Not proper ROC curve corresponding to the expression of gene $\mathbf{n} .3$ in Table 2 (GENE3388X: Immunoglobulin J Chain). Comparison between class A (I4 samples of NBC) and class B (20 heterogeneous lymphomas, including 9 FL and II CLL samples). Hst = Highly stimulated NBC; SSt= Slightly or not stimulated NBC (Table I). NBC samples are numbered according to Table I. 


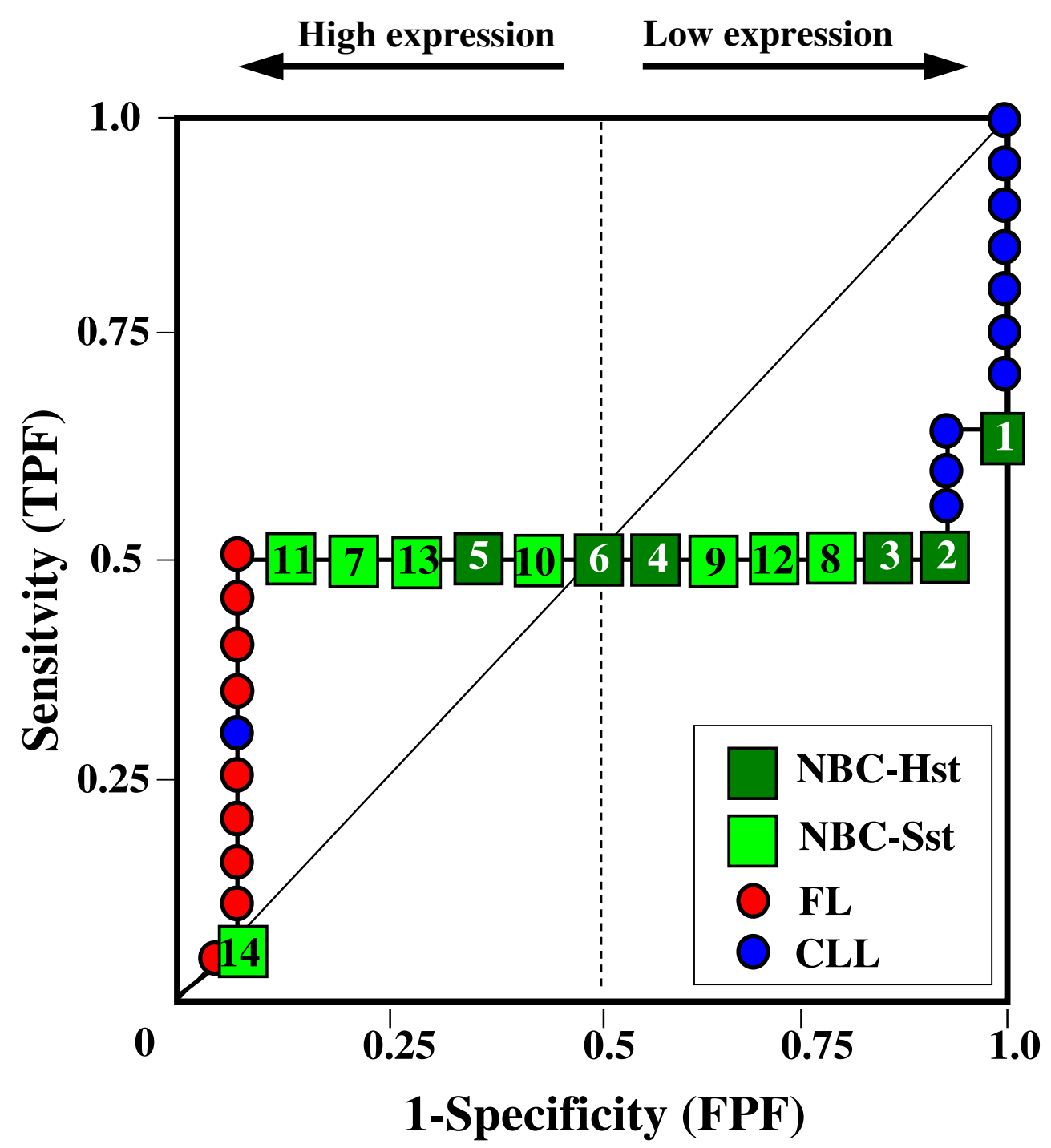

FL CLL

High expression

Low expression

\begin{tabular}{|c|c|}
\hline 9 & 1 \\
\hline 0 & 10 \\
\hline
\end{tabular}

$P<0.001$

Figure 6

Not proper ROC curve corresponding to the expression of gene n. 4 in Table 2 (GENE3323X: BCL7A). Comparison between class A (I4 samples of NBC) and class B (20 heterogeneous lymphomas, including 9 FL and II CLL samples). Hst $=$ Highly stimulated NBC; SSt= Slightly or not stimulated NBC (Table I). NBC samples are numbered according to Table I. 

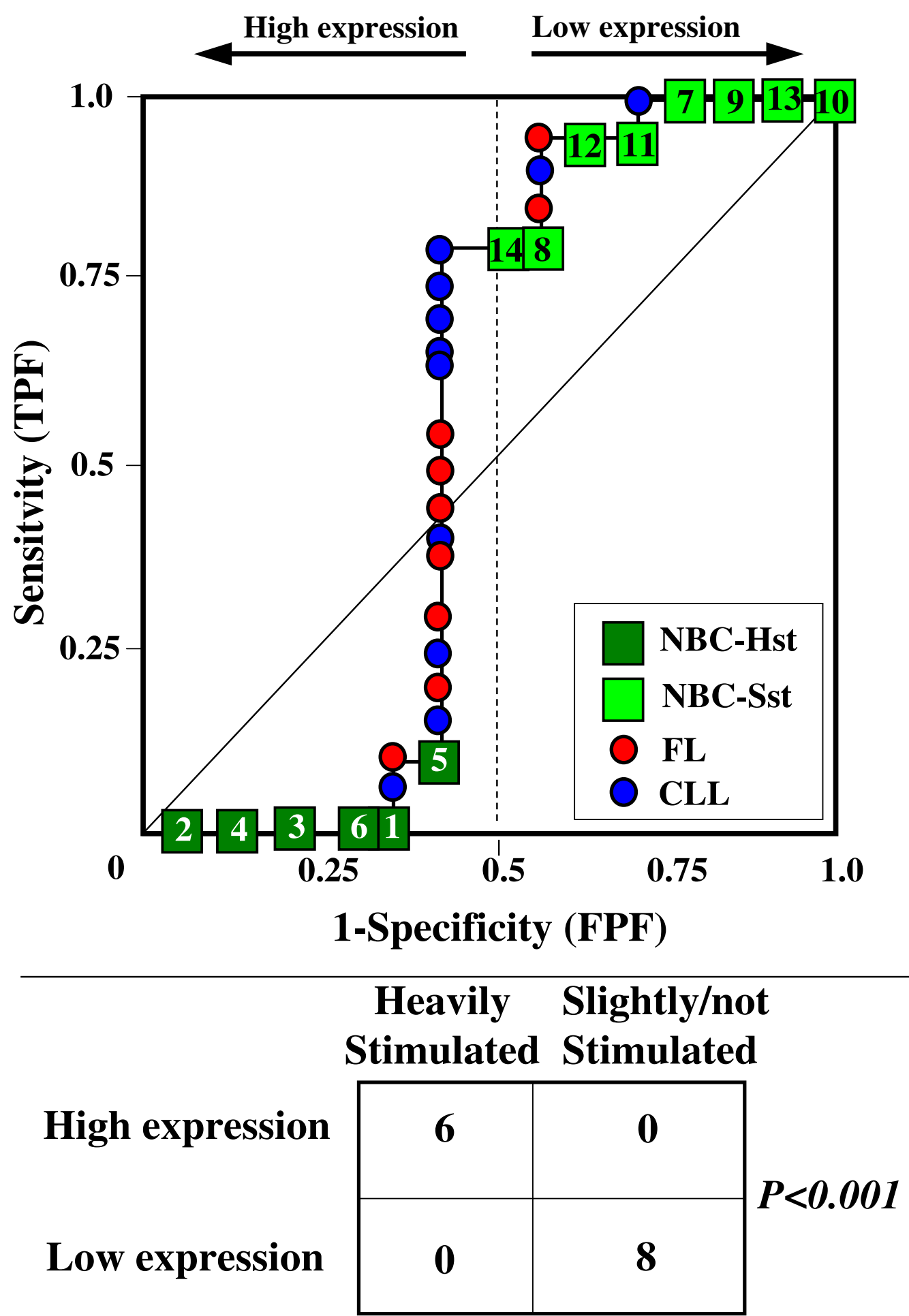

Figure 7

Not proper ROC curve corresponding to the expression of gene n. 5 in Table 2 (GENE3407X: Histone deacetylase 3). Comparison between class A (I4 samples of NBC) and class B (20 heterogeneous lymphomas, including 9 FL and II CLL samples). Hst = Highly stimulated NBC; SSt = Slightly or not stimulated NBC (Table I). NBC samples are numbered according to Table I. 

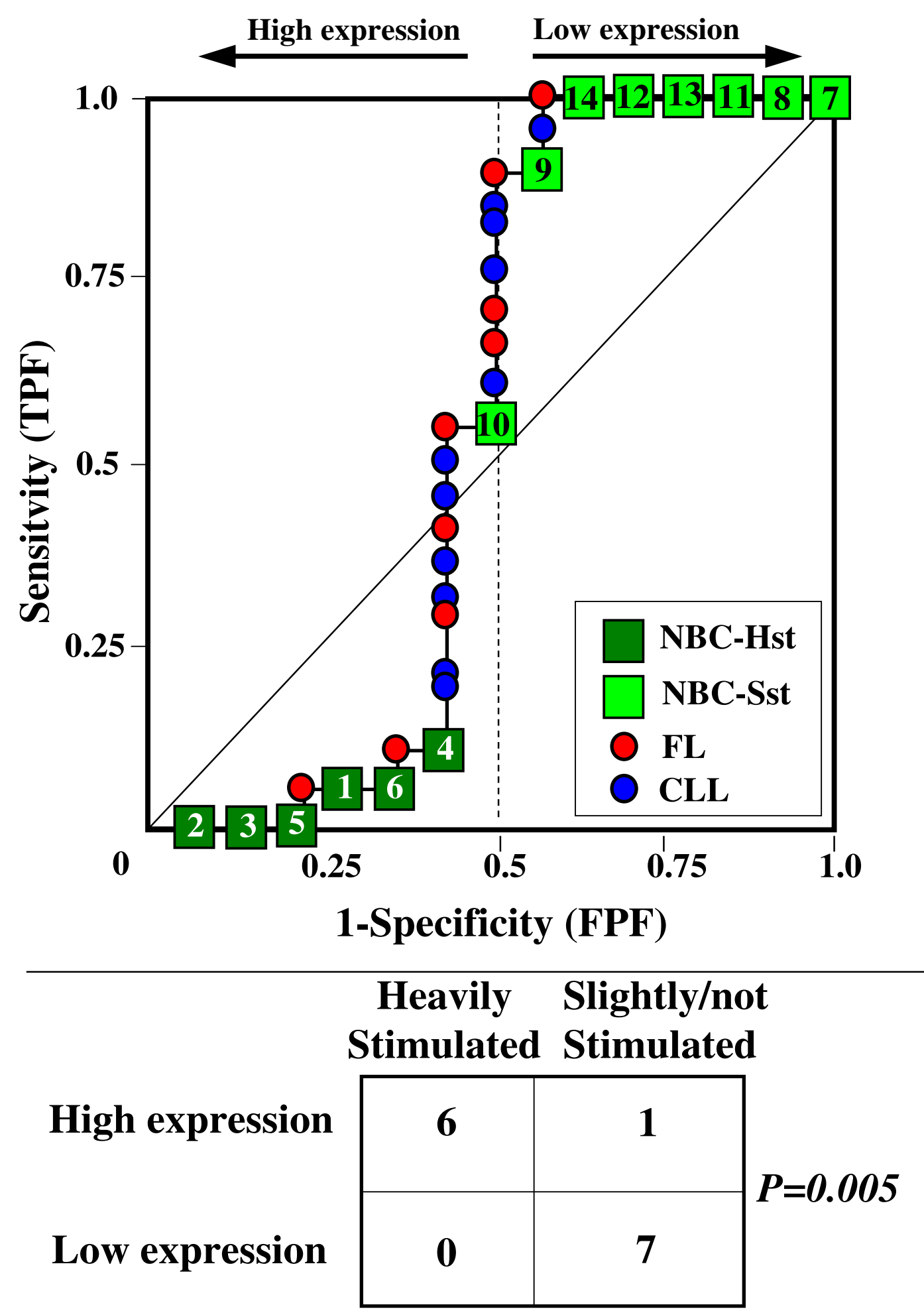

Figure 8

Not proper ROC curve corresponding to the expression of gene n. 6 in Table 2 (GENE75X: VRK2 kinase). Comparison between class A ( I 4 samples of NBC) and class B (20 heterogeneous lymphomas, including 9 FL and I I CLL samples). $\mathrm{Hst}=$ Highly stimulated NBC; SSt= Slightly or not stimulated NBC (Table I). NBC samples are numbered according to Table I. 


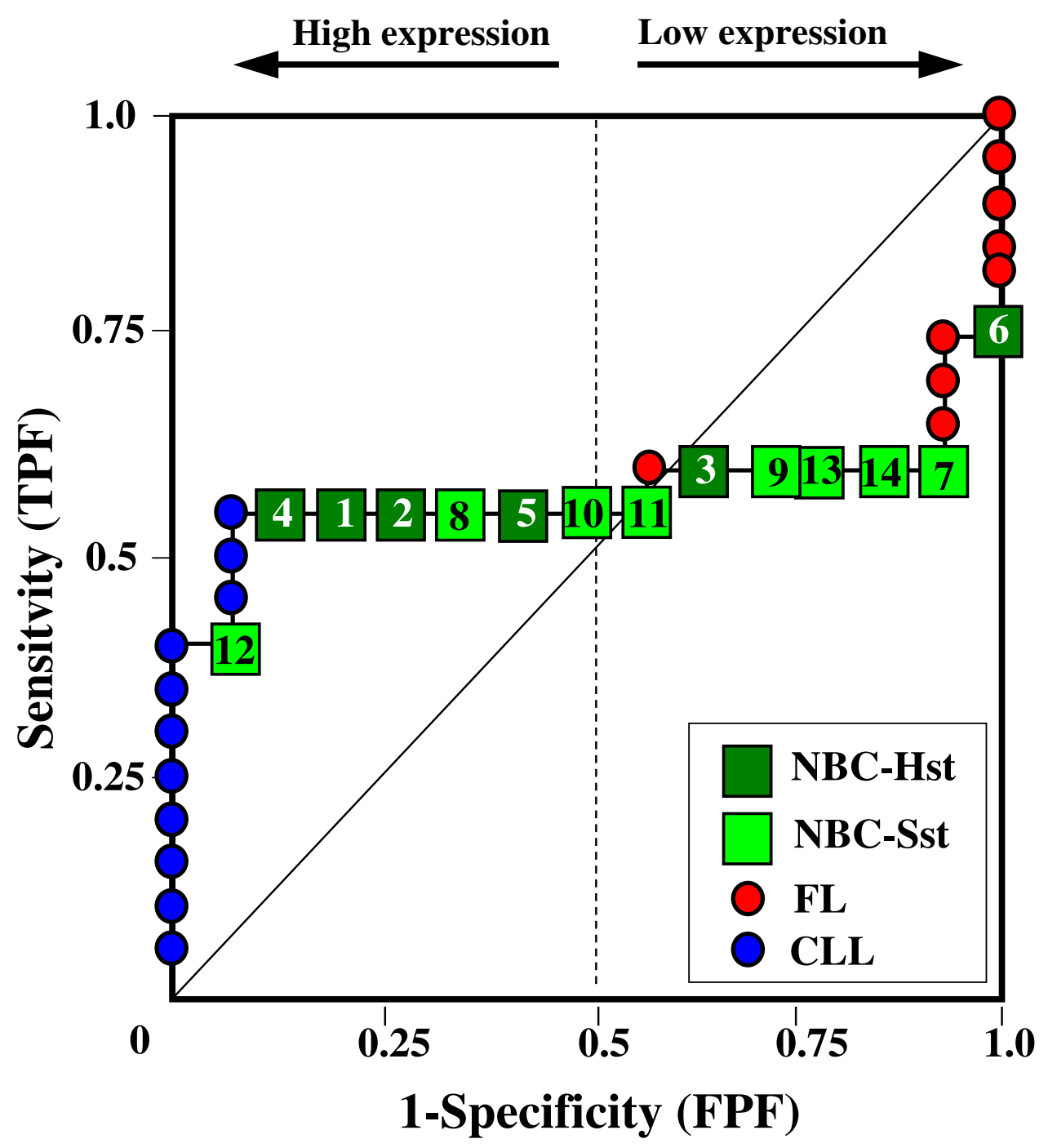

\section{FL CLL}

High expression

Low expression

\begin{tabular}{|c|c|}
\hline 0 & 11 \\
\hline 9 & 0 \\
\hline
\end{tabular}

\section{$P<0.001$}

Figure 9

Not proper ROC curve corresponding to the expression of gene $n .7$ in Table 2 (GENEI I 4 I X: MAPKKK5).

Comparison between class A (I4 samples of NBC) and class B (20 heterogeneous lymphomas, including $9 \mathrm{FL}$ and II CLL samples). Hst = Highly stimulated NBC; SSt= Slightly or not stimulated NBC (Table I). NBC samples are numbered according to Table I. 


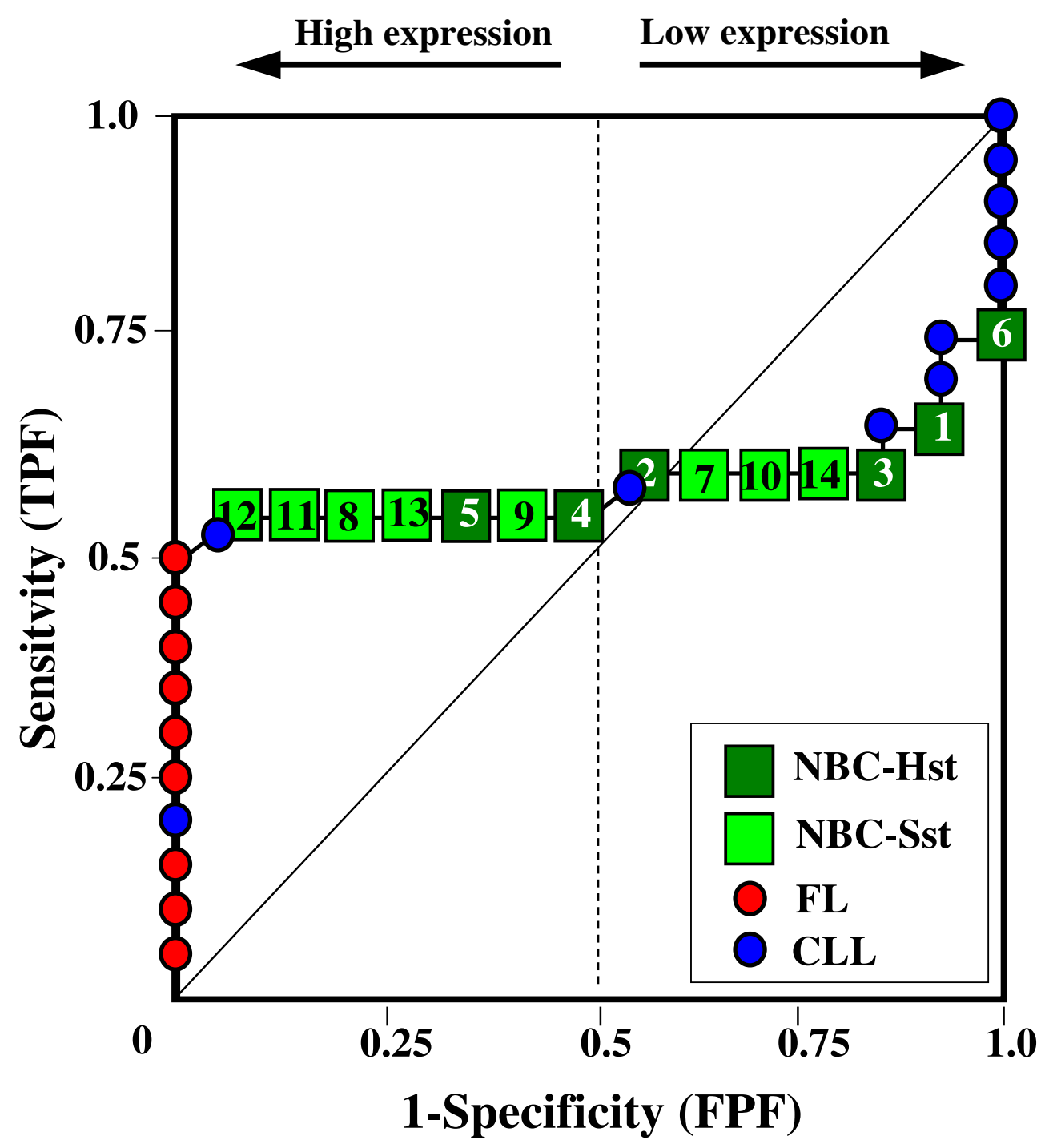

FL CLL

High expression

Low expression

\begin{tabular}{|l|l|}
\hline 9 & 2 \\
\hline 0 & 9 \\
\hline
\end{tabular}

$P<0.001$

Figure 10

Not proper ROC curve corresponding to the expression of gene $n .8$ in Table 2 (GENEI8I 7X: BL34). Comparison between class A (I4 samples of NBC) and class B (20 heterogeneous lymphomas, including 9 FL and II CLL samples). Hst = Highly stimulated NBC; SSt= Slightly or not stimulated NBC (Table I). NBC samples are numbered according to Table I. 


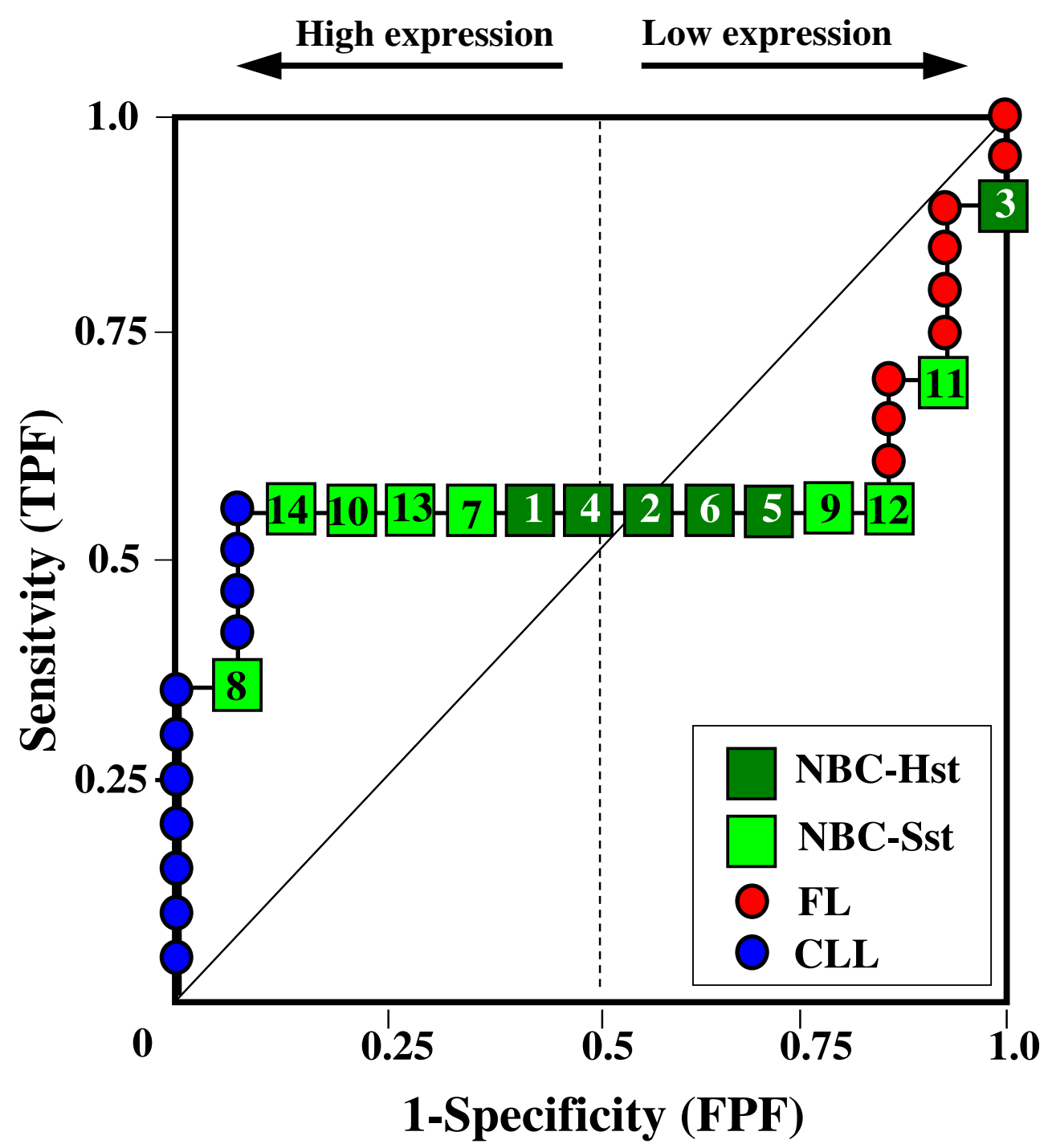

FL CLL

High expression

Low expression

\begin{tabular}{|c|c|}
\hline 0 & 11 \\
\hline 9 & 0 \\
\hline
\end{tabular}

$P<0.001$

Figure II

Not proper ROC curve corresponding to the expression of gene n. 9 in Table 2 (GENE2395X: unknown). Comparison between class A ( I 4 samples of NBC) and class B (20 heterogeneous lymphomas, including 9 FL and I I CLL samples). Hst = Highly stimulated NBC; SSt= Slightly or not stimulated NBC (Table I). NBC samples are numbered according to Table I. 


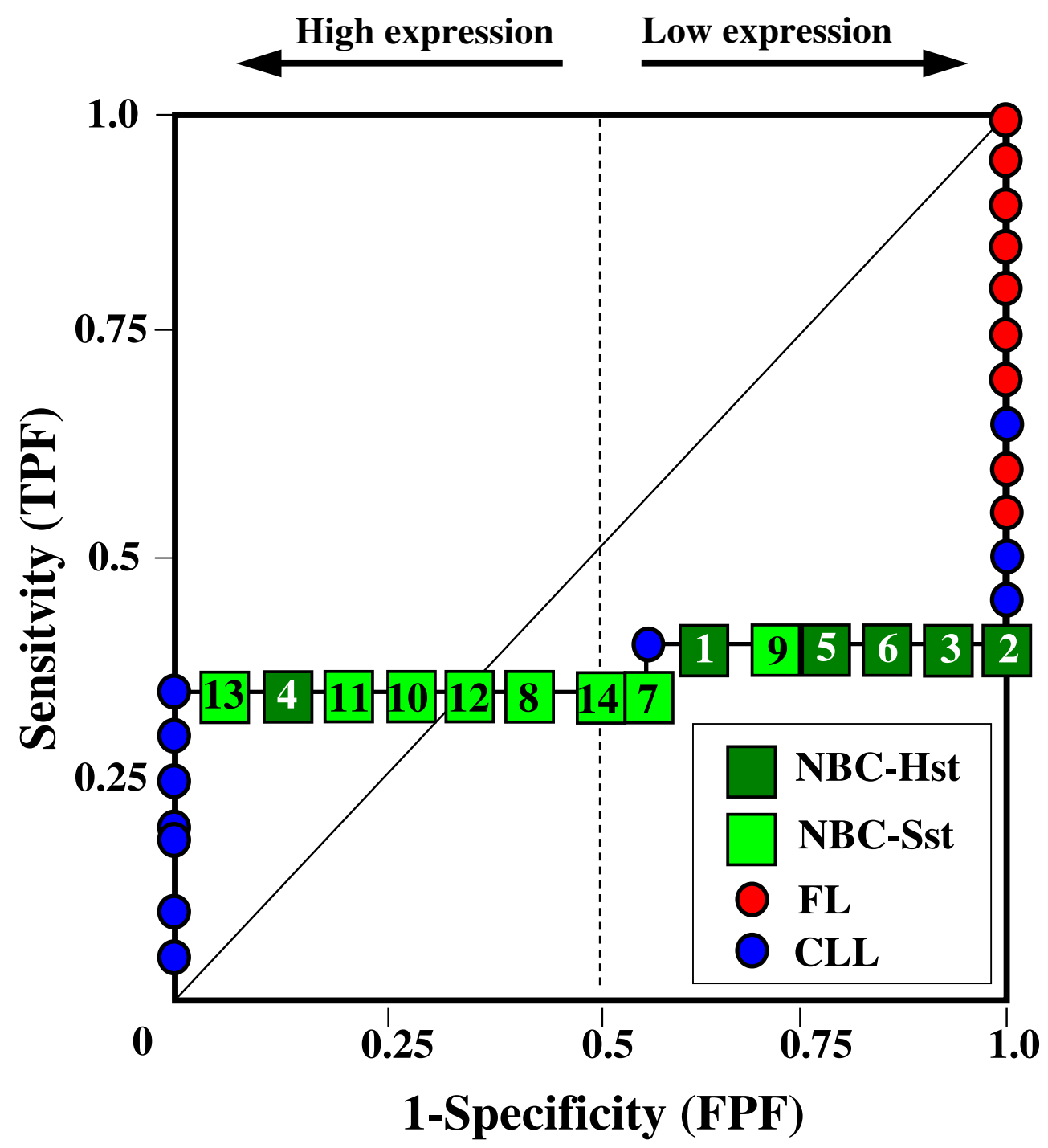

FL CLL

High expression

Low expression

\begin{tabular}{|l|l|}
\hline 0 & 7 \\
\hline 9 & 4 \\
\hline
\end{tabular}

$P=0.005$

Figure 12

Not proper ROC curve corresponding to the expression of gene n. 10 in Table 2 (GENE2696X: unknown). Comparison between class A ( 4 samples of NBC) and class B (20 heterogeneous lymphomas, including 9 FL and II CLL samples). Hst = Highly stimulated NBC; SSt= Slightly or not stimulated NBC (Table I). NBC samples are numbered according to Table I. 

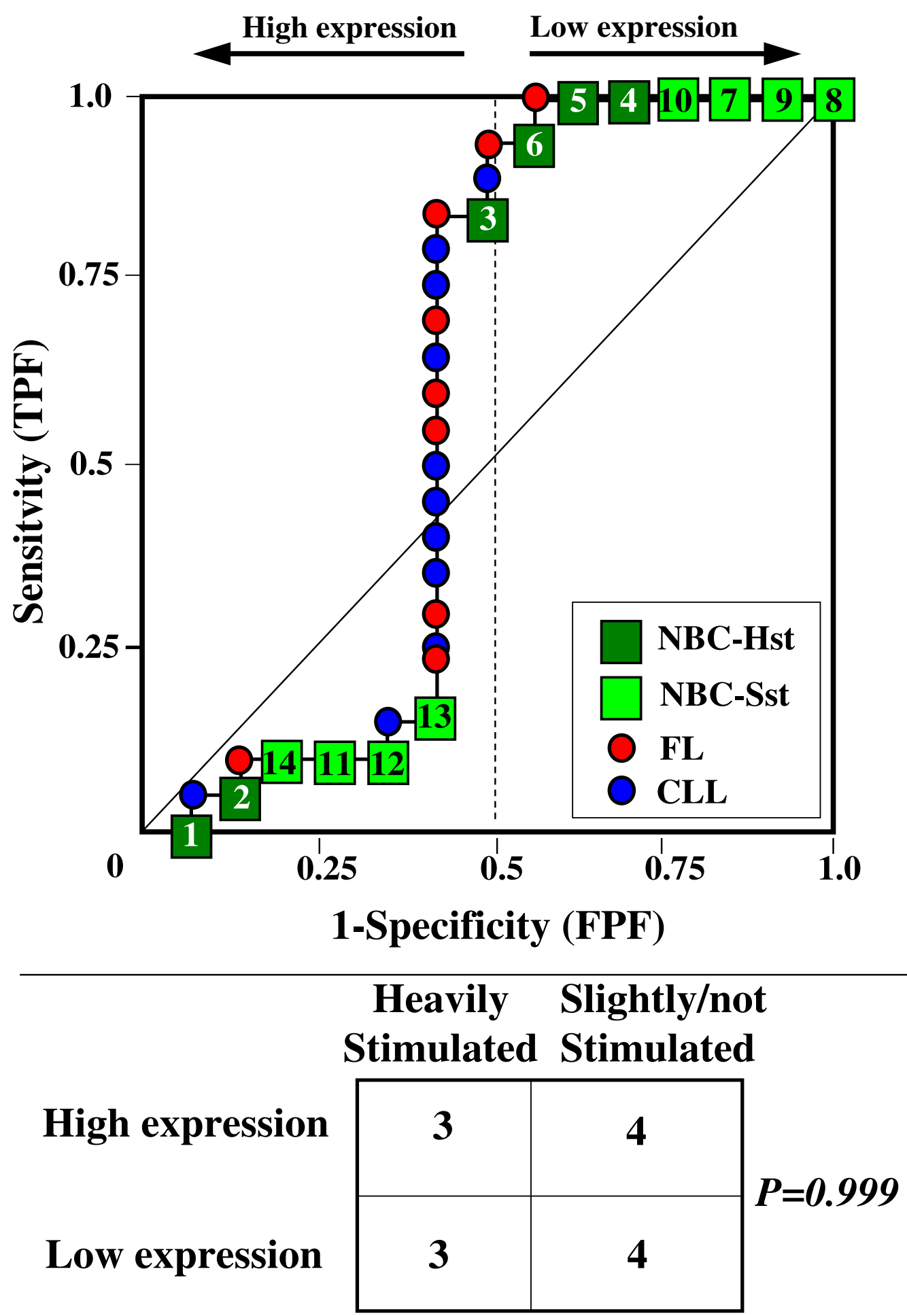

Figure 13

Not proper ROC curve corresponding to the expression of gene $n$. I I in Table 2 (GENE352 IX: Similar to KIAA0050). Comparison between class A (I 4 samples of NBC) and class B (20 heterogeneous lymphomas, including $9 \mathrm{FL}$ and I I CLL samples). Hst = Highly stimulated NBC; SSt= Slightly or not stimulated NBC (Table I). NBC samples are numbered according to Table I. 

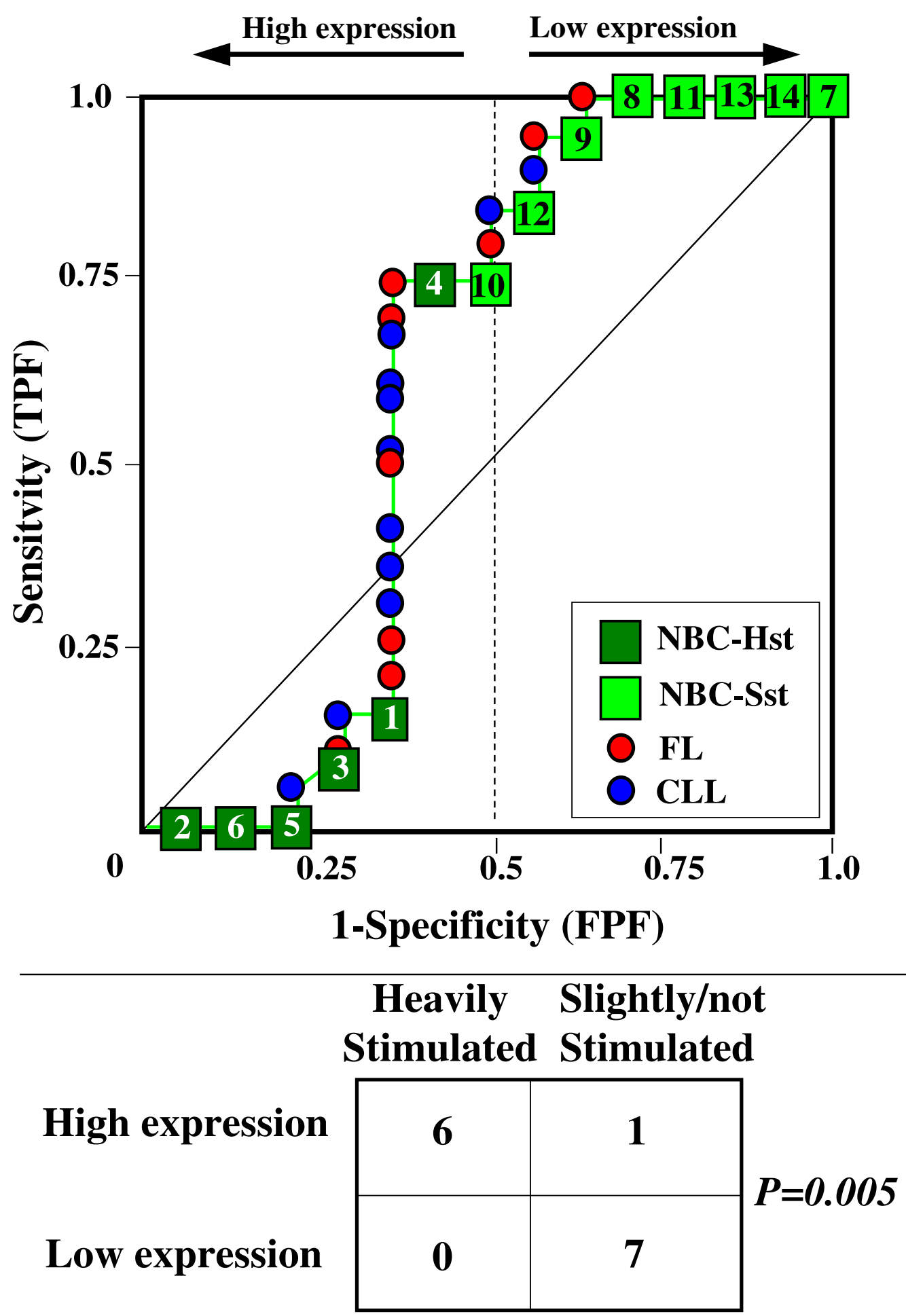

Figure 14

Not proper ROC curve corresponding to the expression of gene $n$. I 2 in Table 2 (GENE74X: VRK2 kinase). Comparison between class A (I4 samples of NBC) and class B (20 heterogeneous lymphomas, including 9 FL and II CLL samples). Hst $=$ Highly stimulated NBC; SSt= Slightly or not stimulated NBC (Table I). NBC samples are numbered according to Table I. 


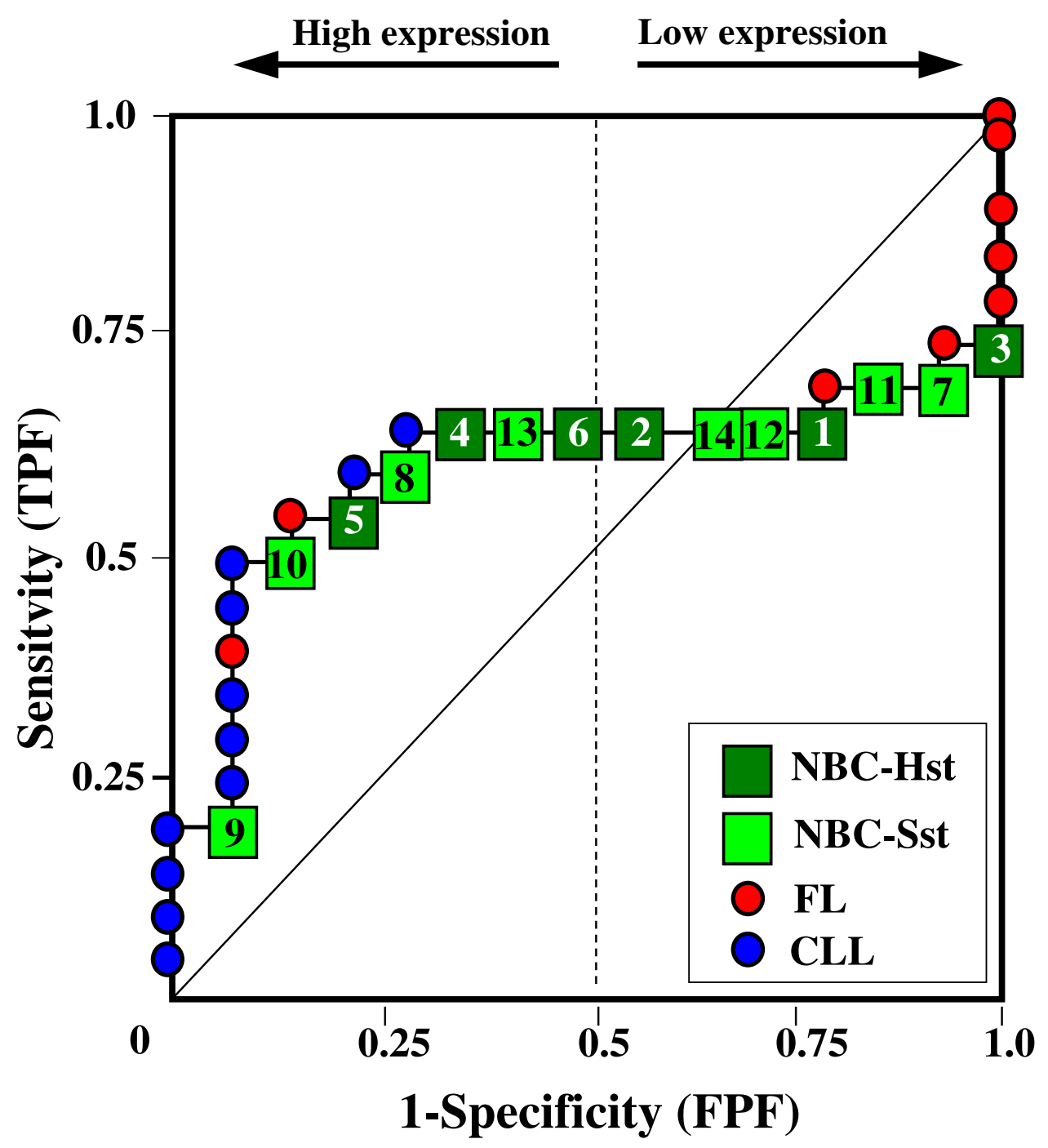

\section{FL CLL}

High expression

Low expression

\begin{tabular}{|c|c|}
\hline 7 & 0 \\
\hline 2 & 11 \\
\hline
\end{tabular}

\section{$P<0.001$}

Figure 15

Not proper ROC curve corresponding to the expression of gene $n .13$ in Table 2 (GENE2287X: MRC OX-2). Comparison between class A ( 14 samples of NBC) and class B (20 heterogeneous lymphomas, including $9 \mathrm{FL}$ and II CLL samples). Hst = Highly stimulated NBC; SSt= Slightly or not stimulated NBC (Table I). NBC samples are numbered according to Table I. 


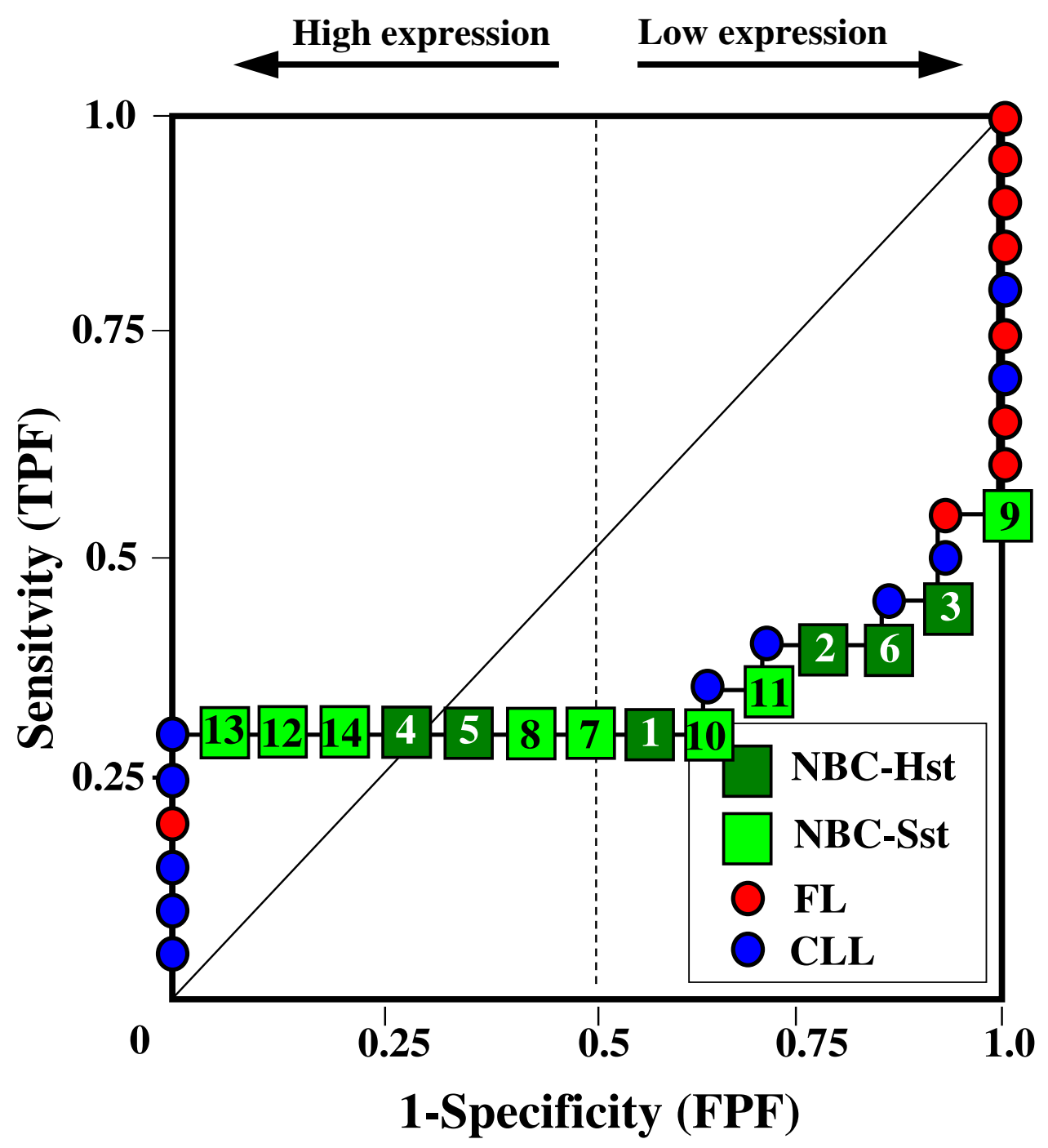

\section{FL CLL}

High expression

Low expression

\begin{tabular}{|c|c|}
\hline 1 & 5 \\
\hline 8 & 6 \\
\hline
\end{tabular}

Figure 16

Not proper ROC curve corresponding to the expression of gene n. I4 in Table 2 (GENE354IX: Unknown). Comparison between class A ( 4 samples of NBC) and class B (20 heterogeneous lymphomas, including 9 FL and II CLL samples). Hst $=$ Highly stimulated NBC; SSt= Slightly or not stimulated NBC (Table I). NBC samples are numbered according to Table I. 

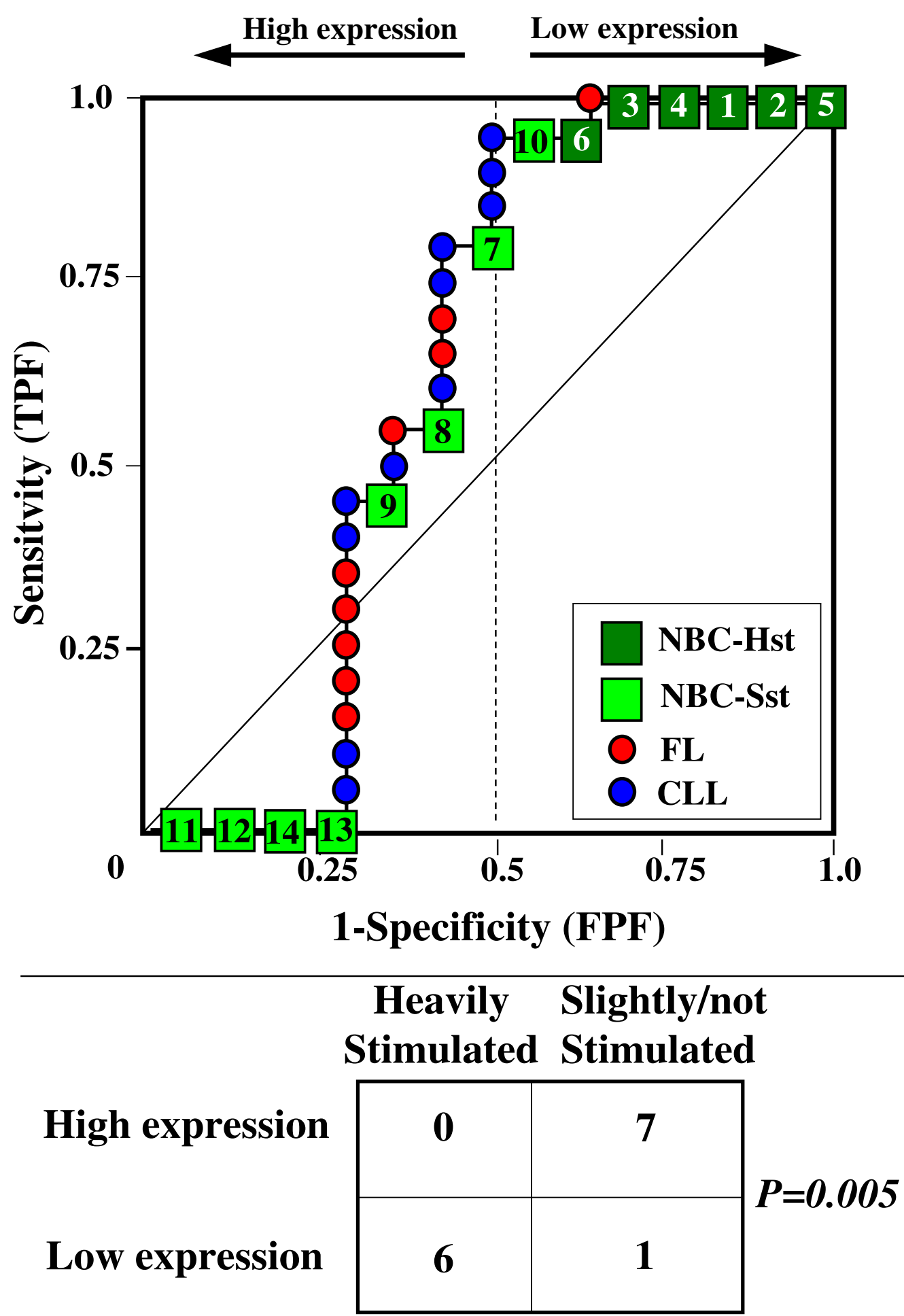

Figure I 7

Not proper ROC curve corresponding to the expression of gene n. I 5 in Table 2 (GENEI362X: Syndecan-2). Comparison between class A (I4 samples of NBC) and class B (20 heterogeneous lymphomas, including 9 FL and II CLL samples). Hst $=$ Highly stimulated NBC; SSt= Slightly or not stimulated NBC (Table I). NBC samples are numbered according to Table I. 

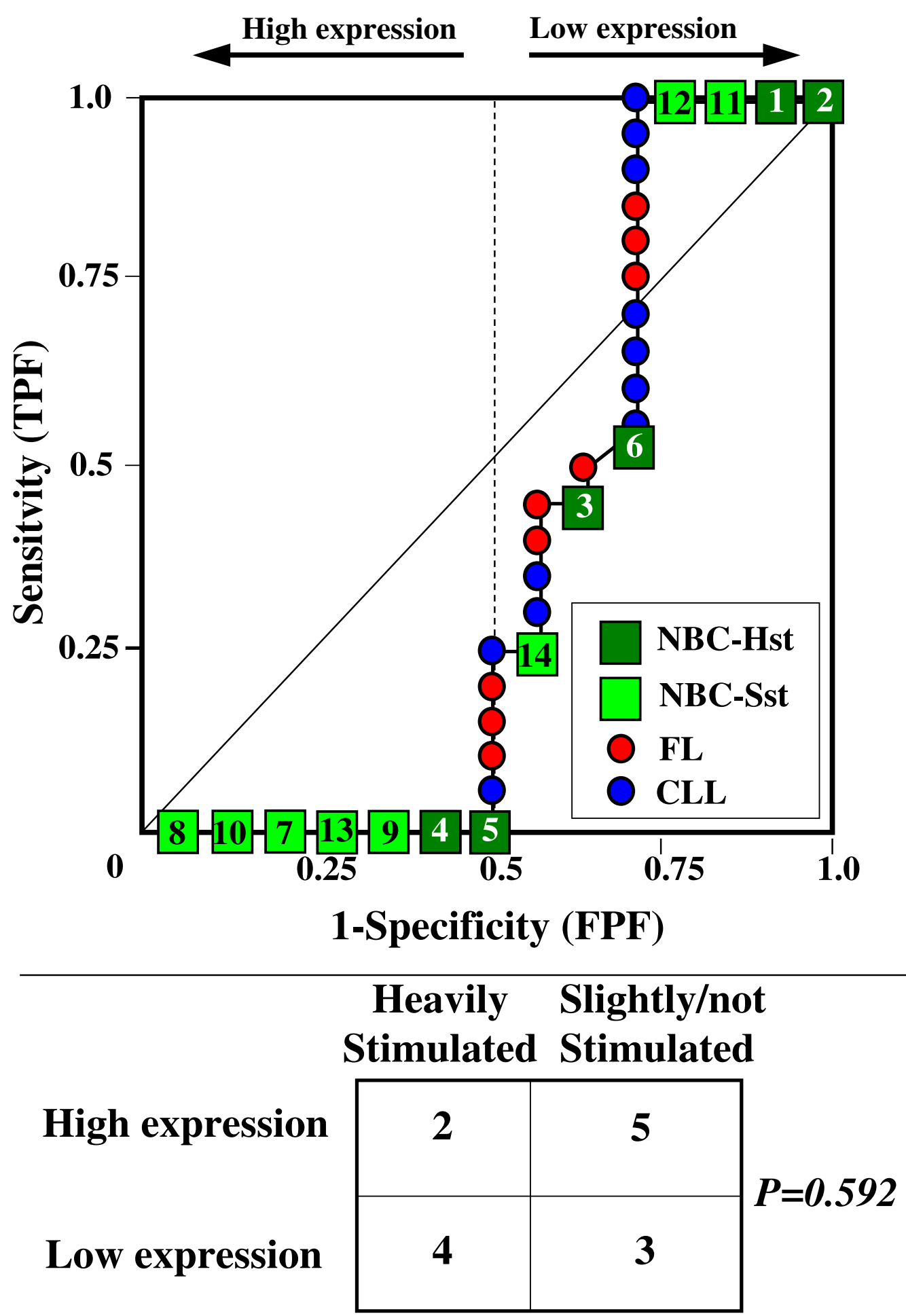

Figure 18

Not proper ROC curve corresponding to the expression of gene n. 16 in Table 2 (GENE2673X: Unknown). Comparison between class A (I 4 samples of NBC) and class B (20 heterogeneous lymphomas, including 9 FL and II CLL samples). Hst $=$ Highly stimulated NBC; SSt= Slightly or not stimulated NBC (Table I). NBC samples are numbered according to Table I. 
with no error for Figure 7 (corresponding to the expression of Histone deacetylase, gene n. 5 in Table 2), 1 error for Figures 8, 14 (both corresponding to clones of VRK2 kinase, genes n. 6 and n. 12, respectively) and 17 (corresponding to Syndecan-2, gene n. 15). All the remaining 10 genes in Figure 3 corresponded to inversely-sigmoid shaped curves and they allowed to separate the two hidden subclasses within class B (FL and CLL), with the only exception of Figure 16 (gene n.14, unknown function). In more detail, the identification of the two hidden subclasses was made with no error in 2 cases (Figure 9, corresponding to gene MAPKKK5, n. 7, and Figure 11, gene n. 9 with unknown function), with 1 error in 3 cases (Figure 3 and Figure 5, corresponding to two clones of Immunoglobulin J chain, genes n. 1 and n.3, respectively, and Figure 6, BCL7A, gene n. 4), with 2 errors in 3 cases (Figure 4, gene n. 2, Immunoglobulin J chain; Figure 10, gene n. 8, BL34; Figure 15, gene n. 13, MRC OX-2), and 4 errors in 1 case (Figure 12, gene n. 10, unknown function). In summary, only 3 out of 16 ROC curves (Figures 13, 16 and 18) were not associated with the presence of $a$ priori known hidden subclasses.

Figure 19 (A-C) shows the results of the analysis of simulated data sets, reporting, for comparison purposes, the performance of $A B C R, T N R C$ and the commonly used $A U C$ statistic. As expected, for all the considered statistics FDR tended to decrease by increasing the sample size, the mean difference $(M D)$ between groups and the number of selected genes. The performance of $A B C R$ and $A U C$ was similar in each analysis, while FDR estimates were systematically higher for TNRC than for the other two statistics. Finally, Figure 20 shows the expected values and variance for the two new proposed ROC parameters $(A B C R$, panel $A$, and TNRC, panel B) estimated under the null hypothesis by $10^{4}$ simulations based on a random permutation analysis. For both statistics the estimated expected value tended to the theoretical one (i.e., 0 in both cases) increasing the sample size, while the variance tended to rapidly decrease, indicating that both $A B C R$ and TNRC are asymptotically unbiased and consistent estimators.

\section{Discussion}

In this paper we have illustrated a new feature selection method using a combination of standard (AUC) and new (ABCR and TNRC) statistical tools based on ROC curves properties. In particular, $A B C R$ represents a new comprehensive test to identify both proper and not proper ROC curves. Because $A B C R$ is a measure of the distance between the cumulative distributions of the two classes under study (as demonstrated in the Material and Methods section), it may be used to potentially identify any kind of differentially expressed genes. AUC represents a well known useful tool to identify under- and overexpressed genes in the comparison between two classes
$[4,7,8,12]$, while TNRC represents a new tool to specifically identify NPRC. As illustrated in Figure 2A, where genes selected by $A B C R$ were separated on the basis of $A U C$ and TNRC values, all the genes identified by TNRC tended to escape feature selection based on AUC and vice versa. This behavior was also confirmed when AUC was replaced by $t$ statistic, another standard feature selection tool $[3,4]$, and when two different thresholds for FDR were used (10\% and $20 \%$, respectively). These results strongly point out that TNRC can identify differentially expressed genes that are hardly identifiable by standard statistical tools.

The large majority of genes selected by $A B C R$ were identifiable by AUC or $t$ statistic and, accordingly, they resulted either under- or over-expressed in lymphoma cells compared with NBC. NPRC represented a very small fraction of the selected genes. This finding might be due to the fact that TNRC statistic tends to identify gene expression profiles that are different in two or more subclasses within one class compared with another, a condition that may be quite rare in real data. However, as indicated by results of simulation analysis (Figure 19) the main limit of TNRC is probably its low statistical power. Simulation analysis was based on a very simplified scenario, because data were generated from a few variety of statistical distributions (namely: normal and bi-normal functions, with similar variance and different means) and the correlation between gene expression profiles was not taken into account. However, in spite of such limitations, the comparison between $T N R C, A B C R$ and AUC clearly indicated a poor performance of TNRC compared with the two latter statistics (Figure 19). As a consequence, in microarray experiments with small sample size TNRC can probably recover only a minor proportion of differentially expressed genes that have escaped standard feature selection methods. However, as illustrated in Figure 2A and 2B, TNRC represents a complementary tool for microarray data that may supplement information from standard statistical analysis. Moreover, the rapid improvements in microarray technology and the consequent availability of chips with a low cost and a high quality might allow a very extensive application of TNRC method in the next future.

We have arbitrarily chosen the conventional threshold of $p=0.05$ to separate different kinds of gene expression profiles (Figure $2 \mathrm{~A}$ and $2 \mathrm{~B}$ ). It is evident from figure $2 \mathrm{~A}$, where only ROC parameters were used, that varying the selected thresholds, almost all unselected genes may have been included in one out of the three considered categories (namely: under-expressed, over-expressed and corresponding to NPRC, respectively). Using $t$ statistic in place of AUC (Figure 2B) a less clear separation between such genes was obtained, leaving a higher number of expression profiles as not classified. In particular, some genes, 
A)

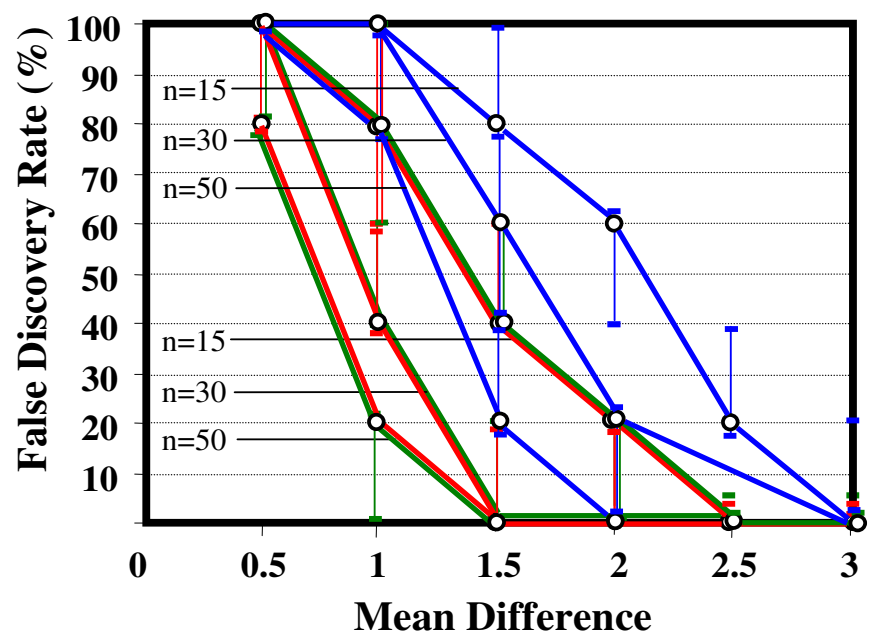

B)

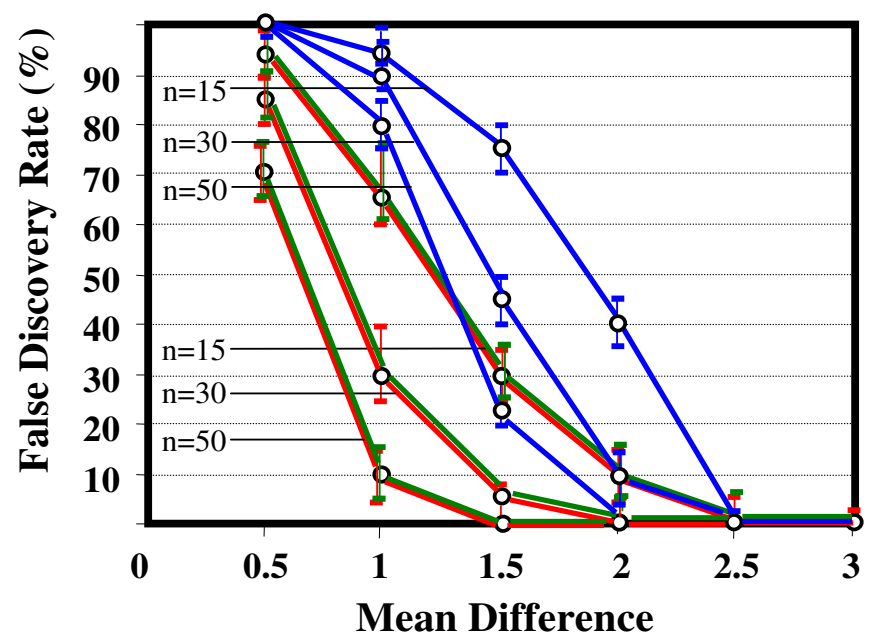

C)

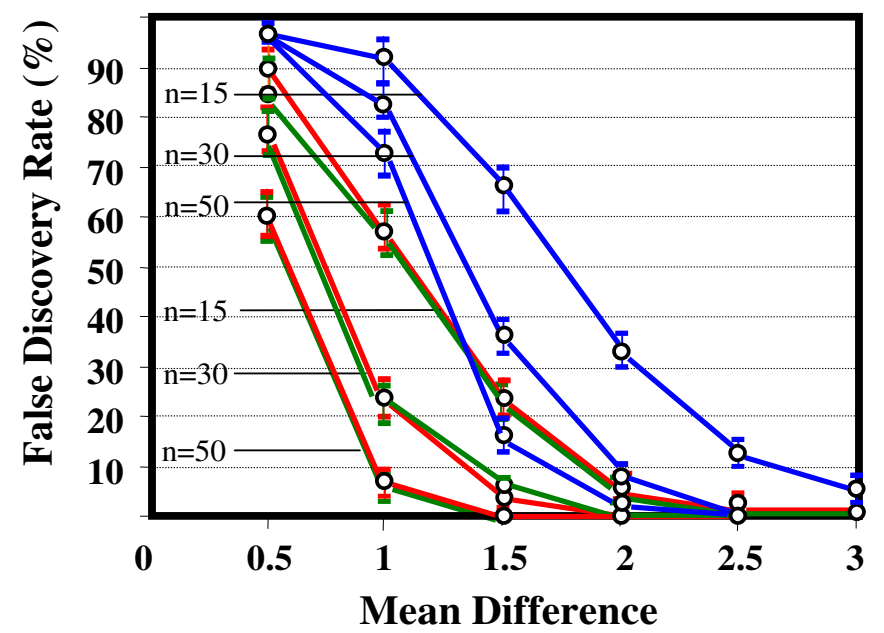

Figure 19

False Discovery Rate of ABCR (green line), TNRC (blue line) and AUC (red line) as a function of the mean difference between class, the sample size in each class and the number $\mathbf{N}$ of selected genes. Median and interquartile range are displayed. Panel $A: N=5$; panel $B: N=20$; Panel $C: N=50$. 
A)
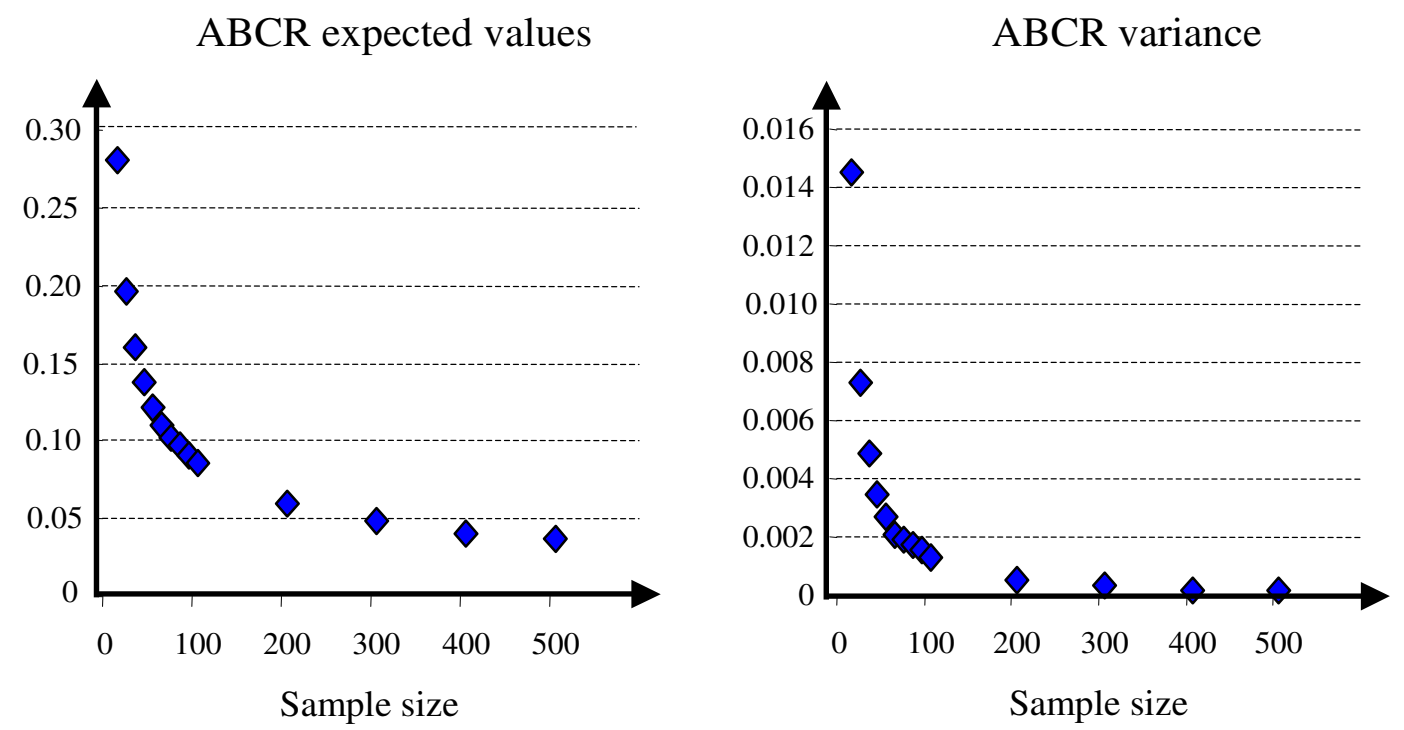

\section{B)}

TNRC expected values

TNRC variance

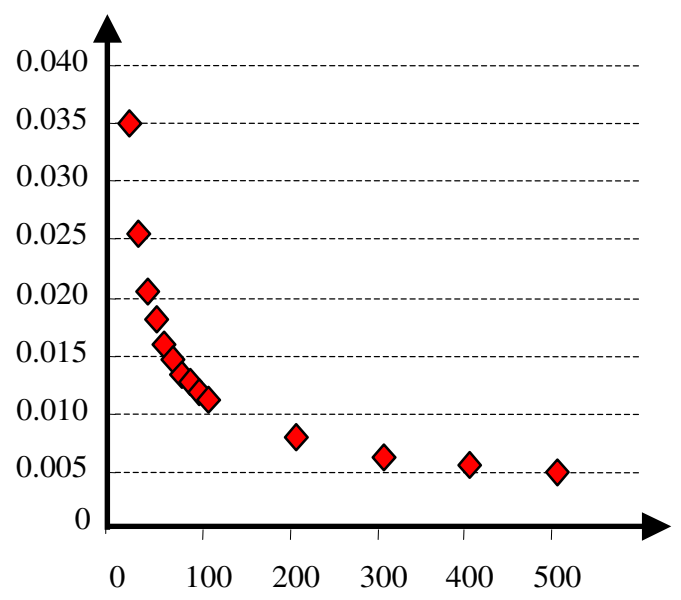

Sample size

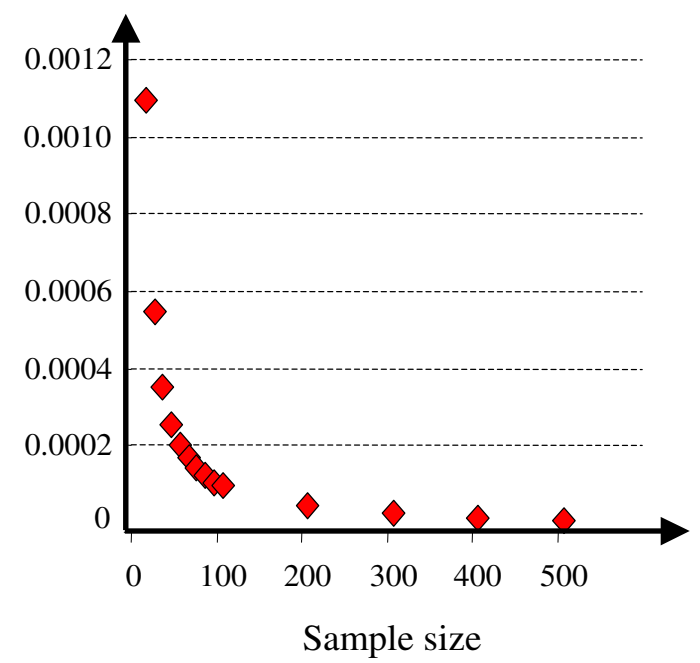

Figure 20

Mean and variance estimates for $A B C R$ and TNRC under the null hypothesis as a function of the number of samples in each class (equal sample size). Each estimate was obtained from $10^{4}$ random permutations. 
corresponding to the central low region of the plot (empty circles), showed low values of both TNRC and $t$ statistics. This finding is not surprising, because such genes were selected on the basis of $A B C R$ statistic, which may take high values even in the presence of a small difference between mean values in the two classes that cause $t$ statistic to approach zero, a phenomenon that may be due to one or more outliers.

Our results confirmed the hypothesis that NPRC (identifiable by TNRC) may correspond to genes, whose expression profile is influenced by the presence of hidden subgroups in either class. In particular, when applied to the comparison between the semi-artificial class $\mathrm{B}$, which included FL and CLL samples, and the class $\mathrm{A}$, which included 14 NBC ( 6 heavily and 8 slightly or not stimulated samples), 13 out of 16 selected genes were able to separate almost perfectly the two hidden subgroups within either one class. In particular, the first three selected profiles in Table 2, corresponding to three clones of the same gene (Immunoglobulin J chain), highlighted the over-expression of all FL samples and the under-expression of all CLL samples (as indicated by the inversely-sigmoid shaped curves in Figure 3, 4 and 5), with only one exception, i.e., a sample of over-expressed CLL. Interestingly, this sample was the same in the three clones (namely "CLL-52" in the original paper) [11]. J chain is a 137-amino acid protein that is synthesized in B lymphocytes and subserves 2 known functions: linking immunoglobulin monomers (IgM to pentamers, IgA to dimers) and binding polymeric immunoglobulin to secretory component [14]. Differential expression of the Immunoglobulin $J$ chain gene in FL vs B-CLL has not been reported so far and its functional significance is unknown. The possibility that our findings reflect a statistical artifact is made unlikely by the concordant results obtained from the analysis of three different clones of the gene (Table 2). Further studies will help to better define this issue. Among the sigmoid shaped curves, which indicate the presence of two hidden sub-classes within NBC samples, the gene with the highest TNRC value was indicated as Gene3407X (n. 5 in Table 2) and corresponded to the Histone deacetylase 3. The corresponding ROC plot (Figure 7) allowed to perfectly separate Hst from Sst cells. Histone deacetylase 3 (HDAC3) shares functional features with HDAC1 and HDAC2. These include deacetylation of histone substrates, promoter targeted transcriptional repression and physical association with the DNA binding factor YY1 [15]. HDCA3 forms a stable complex with nuclear receptor corepressor ( $\mathrm{N}-\mathrm{CoR}$ ) and silencing mediator of retinoic and thyroid receptors (SMRT). Beside to the direct effect on histone deacetylation, the HDAC3-N-CoR complex can exert broader functions in regulating chromatin structure. Aberrant expression and/or localization of
HDCA3 have been reported in various solid tumors and myeloid leukemia [15].

TNRC is a supervised method of statistical analysis that, as illustrated above, may help in the identification of hidden subgroups, a task in general performed by unsupervised clustering. This latter technique has been proven to be very useful in microarray data analysis [1], because it may exploit the correlation between different gene expressions, and may identify genes that are likely to escape supervised feature selection, including TNRC and standard analysis based on AUC or $t$ values. In particular, a different expression profile within a very small sub-class (e.g., two or three samples) is in general hardly identifiable by supervised tests, due to their low statistical power in the presence of small sample size in either one class. Conversely, unsupervised methods tend to generate false clusters even in the presence of random values from uniform probability functions, a behavior that probably represents the major limit of such technique. Moreover, single expression profiles corresponding to NPRC may completely escape unsupervised selection method if they are weakly or not correlated to other gene expressions, but they are potentially identifiable by TNRC. Further studies are needed to find suitable strategies to combine unsupervised methods with supervised techniques, including our proposed approach, in microarray data analysis. Finally, the possible use of the new proposed statistics, and in particular of $A B C R$, to select genes useful for classification methods [16] should also be explored.

\section{Conclusion}

In this paper we have illustrated a new approach for feature selection in microarray data analysis based on a combination of new and standard statistical tools exploiting the properties of ROC curves. Our method may identify both proper ROC plots, using the conventional AUC statistic, and NPRC, corresponding to high values of the new proposed TNRC parameter. AUC is a well known useful tool to identify over- and under-expressed genes, while $T N R C$ can identify differentially expressed genes that tend to escape standard statistical analysis. We have shown that a simple visual inspection at the plot of a NPRC, selected by TNRC, may allow to identify hidden subclasses with potential clinical and biological insight. For these reasons, our results indicate that NPRC represent a new flexible and useful tool for the analysis of gene expression profiles from microarray experiments.

\section{Methods \\ Data sets}

We applied our new method for feature selection both to real and to simulated data sets. We selected a set of real data of gene expression by extracting 34 samples from the large data base by Alizadeh and collaborators [11], pub- 
licly available at the following web site address: http:// llmpp.nih.gov/lymphoma/data/figure1/. This database included 4026 gene expression profiles from a variety of 96 samples of lymphomas or non neoplastic cells. We obtained a first group (named "class A") from 14 samples of normal circulating B cells (NBC) that had been stimulated in different ways (Table 1; see also Figure 4 in the original paper). On the basis of such stimulation pattern we defined a priori two major subclasses within class A, i.e., 6 highly stimulated and 8 slightly or not stimulated samples (Table 1). We obtained a second semi-artificial group (named "class B") of 20 heterogeneous lymphomas by pooling 9 samples of follicular lymphomas (FL) and 11 samples of chronic lymphocytic leukemia (CLL). A variable proportion of missing values for gene expression was present in each considered group. In particular, in class A the median proportion of missing values was $6.1 \%$ (range: $0.42 \%-31.8 \%$ ), and in class B was $4.7 \%$ (range: $0.17 \%-22.5 \%$ ). We estimated missing data by the method proposed by Troyanskaya and collaborators [17], using $\mathrm{k}=12$ nearest neighbor genes.

We obtained a set of artificial data bases by randomly generating normally distributed data with different means and equal variance in each class or subclass. For instance, we labeled a class of samples as "controls" and a second class of samples as "cases"; we obtained a set of not differentially expressed genes generating similar expression profiles in cases and in controls by randomly extracting simulated values from a normal standard distribution ( mean $=0$ and variance $=1$ ). We obtained another set of over-expressed genes by extracting values from the same distribution, and by adopting different means for cases and controls; the mean difference (MD) between the two classes ranged from 0.5 to 3.0. Finally, we obtained a third set of differentially expressed genes, corresponding to not proper ROC curves, by splitting the cases into two subclasses (one including "under-expressed" values and one including "over-expressed" values, respectively, in comparison with the control class); in such a simulation process, we generated data from normal distributions with different means and equal variance (see as an example, Curve III in Figure 1).

Each simulated data matrix included 4000 genes. We recursively regenerated each artificial data base for 1000 times, allowing the sample size to vary between 15 to 50 in each class and the number of differentially expressed genes from 5 to 50 in each group (class or subclass, where present).

We performed all analyses by an ad hoc program developed in Visual Basic.net academic version (Microsoft.net framework ver. 1.1.4322), available on request. We obtained random numbers for bootstrap procedures and for data sets generation by the RAN1 algorithm [18].

\section{Definition of TNRC and ABCR statistics}

Consider a study involving $n$ subjects, classified by a binary outcome $Y$ taking values in $\{0,1\}$. For example in a case-control study design, individuals with $Y=1$ may be affected by a specific disease, while individuals with $\mathrm{Y}=0$ may be the unaffected controls [5]. Suppose that a variable of interest (e.g., the expression level of a given gene) is measured in all the $n$ subjects of the study. If $n_{0}$ is the number of individuals with $Y=0$, denote with $X_{1}, X_{2}, \ldots$, $X_{n_{0}}$ the values assumed by the variable of interest in this group of subjects; similarly, denote with $W_{1}, W_{2}, \ldots, W_{n_{1}}$ the values measured in the $n_{1}$ individuals with $Y=1$.

The empirical ROC curve can then be defined by considering different threshold values $c$ for the variable of interest and by computing the true and the false positive fractions, denoted by $\operatorname{TPF}(c)$ and $\operatorname{FPF}(c)$, respectively, in the sample at hand [5]. It can be seen that:

$$
\operatorname{TPF}(c)=\frac{\sum_{j=1}^{n_{1}} I\left(W_{j} \geq c\right)}{n_{1}}, \quad \operatorname{PPF}(c)=\frac{\sum_{i=1}^{n_{0}} I\left(X_{i} \geq c\right)}{n_{0}}
$$

where $I$ is the indicator function providing $I\left(X_{i} \geq c\right)=1$ if $X_{i} \geq c$, and $I\left(X_{i} \geq c\right)=0$ otherwise [5]. TPF corresponds to the sensitivity of a diagnostic test, while FPF corresponds to 1 - specificity. Since some of the $X_{i}$ may be equal, let $\left\{c_{1}, \ldots, c_{m_{0}}\right\}$ be the set of the $m_{0}$ different values assumed by $X_{i}$ for $i=1, \ldots, n_{0}$, ordered in a decreasing way $\left(c_{1}>c_{2}>\cdots>c_{m_{0}-1}>c_{m_{0}}\right)$. With these definitions, the ROC curve is given by the two dimensional graph obtained by connecting the points $\left.\operatorname{FPF}\left(c_{k}\right), \operatorname{TPF}\left(c_{k}\right)\right)$ with straight lines, when $k=0,1, \ldots, m_{0}$, being $c_{0}$ any value greater than $X_{i}$ and $W_{j}$ for any $i=1, \ldots, n_{0}$ and any $j=1, \ldots$, $n_{1}$. It can be easily seen that $\left(\operatorname{FPF}\left(c_{0}\right), \operatorname{TPF}\left(c_{0}\right)\right)=(0,0)$ whereas $\left.\operatorname{fPF}\left(c_{m_{0}}\right), \operatorname{TPF}\left(c_{m_{0}}\right)\right)=(1,1)$.

Let $A U C_{k}$ be the partial area under an ROC curve between the consecutive abscissa points $\operatorname{FPF}\left(c_{k-1}\right)$ and $\operatorname{FPF}\left(c_{k}\right)$, for $k$ $=1, \ldots, m_{0}$, computed according to the standard trapezoidal rule. The total area AUC under the ROC curve is then given by 


$$
A U C=\sum_{k=1}^{m_{0}} A U C_{k}=\sum_{k=1}^{m_{0}}\left(\operatorname{TPF}\left(c_{k}\right)+\operatorname{TPF}\left(c_{k-1}\right)\right) \cdot\left(\operatorname{FPF}\left(c_{k}\right)-F P F\left(c_{k-1}\right)\right) / 2
$$

When $\operatorname{TPF}\left(c_{k}\right)=\operatorname{FPF}\left(c_{k}\right)=k / m_{0}$ for $k=0,1, \ldots, m_{0}$, every threshold $c_{k}$ is not able to provide a valid classification for the two groups of subjects, i.e., the class is assigned by chance. In this case we obtain a particular ROC curve, named chance line, corresponding to the rising diagonal, whose partial area $A U C_{k}$ will be denoted by $A_{k}$ and is given by

$$
A_{k}=\frac{2 k-1}{2 m_{0}^{2}}
$$

It should be observed that $A U C=0.5$ for the chance line.

The area AUC under the ROC curve gives a measure of the difference between the two distributions that generated the samples $\left\{X_{i}\right\}$ and $\left\{W_{j}\right\}$. The greater is the value of $A U C$, the higher is the difference between the two distributions [5]. However, in some cases the ROC curve is not proper and crosses the chance line in one or more points. In these cases, even if the value of AUC is close to 0.5, the two distributions can differ significantly.

To recover these situations, the TNRC statistic $(T N R C=$ Test for Not-proper ROC Curves) is introduced, by employing the following definition:

$$
T N R C=\sum_{k=1}^{m_{0}}\left|A U C_{k}-A_{k}\right|-|A U C-0.5|
$$

Since in a proper ROC curve we have $A U C_{k} \geq A_{k}$ for every $k=0,1, \ldots, m_{0}$, equation (1) gives $T N R C=0$. As a special case, this holds also for the chance line.

In addition, it can be easily seen that the value of TNRC is always non negative since

$$
\sum_{k=1}^{m_{0}}\left|A U C_{k}-A_{k}\right| \geq\left|\sum_{k=1}^{m_{0}}\left(A U C_{k}-A_{k}\right)\right|=|A U C-0.5|
$$

The first part of the TNRC statistic corresponds to the area between the ROC curve and the rising diagonal $(A B C R=$ Area Between the Curve and the Rising diagonal):

$$
A B C R=\sum_{k=1}^{m_{0}}\left|A U C_{k}-A_{k}\right|
$$

$A B C R$ is a measure of the distance between the two cumulative distributions $P_{0}(x)$ and $P_{1}(w)$ that generated the samples $\left\{X_{i}\right\}$ and $\left\{W_{j}\right\}$. This can be viewed by considering the theoretical expression of the ROC curve, which is given by $Q_{1}\left(Q_{0}^{-1}(z)\right)$ for $0 \leq z \leq 1$, being $Q_{0}(x)=1$ $P_{0}(x)$ and $Q_{1}(w)=1-P_{1}(w)$ [5]. In this case, $A B C R$ is given by:

$$
\int_{0}^{1}\left|Q_{1}\left(Q_{0}^{-1}(z)\right)-z\right| d z=-\int_{+\infty}^{-\infty}\left|1-P_{1}(x)-1+P_{0}(x)\right| p_{0}(x) d x=\int_{-\infty}^{+\infty}\left|P_{1}(x)-P_{0}(x)\right| p_{0}(x) d x
$$

having performed the change of variable $z=Q_{0}(x)=1$ $P_{0}(x)$. As expected, the term at the right hand side of (3) is just the $L_{1}\left(p_{0}\right)$ distance between the two distributions $P_{0}(x)$ and $P_{1}(w)$, where $p_{0}$ is the probability density of the sample $\left\{X_{i}\right\}$.

The distribution of $A B C R$ and TNRC under the null hypothesis of equal gene expression in the two considered classes was estimated by $10^{4}$ random permutations at different sample size, and sample mean and variance of both estimators were computed.

\section{Feature selection}

As shown in the previous paragraph, the new described ROC parameter $A B C R$ represents a measure of the distance between the distributions of gene expressions in the two considered classes. Then it may be useful to identify differentially expressed genes that may correspond both to proper and to not proper ROC curves. We performed a first step of feature selection by ranking all genes on their values of $A B C R$. The first $k$ genes corresponding to an estimated False Discovery Rate (FDR) of $15 \%$ were retained; the analyses were also repeated using two different FDR thresholds (i.e., $10 \%$ and $20 \%$ ). FDR represents the proportion of gene expression profiles wrongly selected among the $\mathrm{k}$ top ones $[12,19,20]$; we obtained a conservative FDR estimation by 200 random permutations of the labels identifying either one class. Briefly, for each iteration, we computed the number $v$ of values higher than the $A B C R$ value corresponding to the $\mathrm{k}^{\text {th }}$ top selected gene. The mean of $v$ from all permutations divided by $\mathrm{k}$ provided an estimate of FDR $[12,20]$. Finally, we estimated the probability for each gene to be included among the $\mathrm{k}$ ones with the highest $A B C R$ statistic by the method proposed by Pepe and collaborators [8], originally used to account for multiplicity in a similar feature selection task based on another ROC parameter (i.e., pAUC). Briefly, the probability $P_{g}(k)$ of each gene $g$ to be included in such group is [8]:

$$
P_{g}(k)=P[\operatorname{rank}(g) \leq k]
$$


We estimated $P_{g}(k)$ by the bootstrap based on 200 bootstrapped samples, which has the property to acknowledge the complex correlation between gene expression values [8]. We "jittered" each bootstrapped sample adding a randomly generated small number to each gene expression value, to avoid ties that might bias statistical estimates [8]. For this task, random values were extracted from the uniform probability function setting the range of generated values in order to preserve the original rank of not tied values.

The second step of feature selection was based on a standard ROC analysis approach: each gene selected in the previous step was classified as "under-expressed" or "overexpressed" in class B compared with class A, on the basis of the corresponding $A U C$ value (values close to 0 indicating under-expression and values close to 1 indicating over-expression). Moreover, genes were also classified as corresponding to either proper or not proper ROC curves on the basis of the corresponding TNRC value. For both classifications an arbitrary threshold corresponding to the conventional 0.05 unadjusted p value was applied. For the first classification, the threshold value identification was based on the asymptotic normality of AUC and on its relation with the Mann-Whitney $U$ statistic [5,21]. The corresponding critical value for TNRC was obtained by extensive permutations. For comparison purposes, the same analysis was also repeated replacing AUC with the Student's $t$ statistic, which represents another standard tool in supervised analysis of microarrays $[3,4]$. Due to the non normal distribution of most gene expression profiles, which prevents the application of the Student's $t$ distribution tables, statistical significance of $t$ test was assessed by 5000 random permutations.

Finally, in the analysis of simulated data, for each simulation we computed the proportion of proper or uninformative curves included in the first $n$ plots (with $n=$ the number of genes in each simulation, corresponding to the highest TNRC value). Median and interquartile range (IQR) of such proportion, obtained from 1000 simulations, provided a robust estimate of FDR and of its variability, respectively. Finally, by using the same approach, the proportion of any kind of differentially expressed genes and the proportion of genes with different mean value between two classes were used to estimate FDR for $A B C R$ and $A U C$, respectively.

\section{Interpretating the shape of a not proper ROC curve}

We separated the ROC plots identified by TNRC into three categories, on the basis of their shape: a) sigmoid-shaped curves (e.g., Curve III in Figure 1A) that may indicate the presence of a unimodal distribution of expression values in class $\mathrm{B}$ and a bimodal distribution in class A; b) inverse sigmoid-shaped curves (e.g., Curve IV in Figure 1A) that may correspond to a bimodal distribution in class $\mathrm{B}$ and a unimodal distribution in class $\mathrm{A}$; $\mathrm{c}$ ) other differently shaped curves. Furthermore, we arbitrarily split each ROC curve into two parts by a vertical line crossing the centre of the plot (i.e, corresponding to the cut-off with a specificity value $=0.5$ ). In sigmoid-shaped curves, such a cut-off allowed to roughly separate two alleged sub-classes of $\mathrm{NBC}$, i.e., samples with a higher or a lower gene expression in comparison with the median expression value of samples in class B. We evaluated the association between such sub-classes and the stimulation pattern, dichotomized into Hst and Sst, by the Fisher's exact test. Conversely, in inversely sigmoid-shaped curves, such a cut-off allowed to separate two alleged sub-classes of samples among class $\mathrm{B}$, with either over- or under-expression values in comparison with NBC. We also assessed the concordance between such classification and the origin of each sample (FL or CLL) by the Fisher's exact test. The conventional unadjusted p level of 0.05 was used in this analysis.

\section{Authors' contributions}

SP conceived the study, performed all statistical analyses and drafted the manuscript; VP provided biological interpretation of the selected gene expression profiles; MM participated in the design and coordination of the study, and supervised statistical analyses.

\section{Acknowledgements}

This investigation was supported by the Italian Neuroblastoma Foundation (Fondazione Italiana per la Lotta al Neuroblastoma). SP is a recipient of a grant from the Italian Neuroblastoma Foundation.

The authors thank Dr Riccardo Haupt (G. Gaslini Children's Hospital), who revised the manuscript and provided precious advice.

\section{References}

I. Quackenbush J: Microarray analysis and tumor classification. N Engl J Med 2006, 354:2463-2472.

2. Gusnanto A, Calza S, Pawitan Y: Identification of differentially expressed genes and false discovery rate in microarray studies. Curr Opin Lipidol 2007, 18:187-193.

3. Dudoit S, Yang YH, Speed TP, Callow MJ: Statistical methods for identifying differentially expressed genes in replicated cDNA microarray experiments. Statistica Sinica 2002, I 2: I | I-139.

4. Jeffery IB, Higgins DG, Culhane AC: Comparison and evaluation of methods for generating differentially expressed gene lists from microarray data. BMC Bioinformatics 2006, 7:359.

5. Pepe MS: The statistical evaluation of medical tests for classification and prediction Oxford (UK): Oxford University Press; 2003.

6. Parodi S, Muselli M, Fontana V, Bonassi S: ROC curves are a suitable and flexible tool for the analysis of gene expression profiles. Cytogenet Genome Res 2003, 101:90-91.

7. Baker SG: The central role of receiver operating characteristic (ROC) curves in evaluating tests for the early detection of cancer. J Natl Cancer Inst 2003, 95:5 I I-5 I5.

8. Pepe MS, Longton G, Anderson GL, Schummer M: Selecting differentially expressed genes from microarray experiments. Biometrics 2003, 59: 133-142.

9. Metz CE, Herman BA, Shen JH: Maximum likelihood estimation of receiver operating characteristic (ROC) curves from continuously-distributed data. Stat Med 1998, 17:1033-1053. 
10. Lee WC, Hsiao CK: Alternative summary indices for the receiver operating characteristic curve. Epidemiology 1996, 7:605-6II.

II. Alizadeh AA, Eisen MB, Davis RE, Ma C, Lossos IS, Rosenwald A, Boldrick JC, Sabet H, Tran T, Yu X, Powell Jl, Yang L, Marti GE, Moore T, Hudson J Jr, Lu L, Lewis DB, Tibshirani R, Sherlock G, Chan WC, Greiner TC, Weisenburger DD, Armitage JO, Warnke R, Levy R, Wilson W, Grever MR, Byrd JC, Botstein D, Brown PO, et al.: Distinct types of diffuse large B-cell lymphoma identified by gene expression profiling. Nature 2000, 403:503-5II.

12. Tsai CA, Chen Jj: Significance analysis or ROC indices for comparing diagnostic markers: applications to gene microarray data. J Biopharm Stat 2004, 1 4:985- 1003.

13. OMIM, Online Mendelian Inheritance in Man [http:// www.ncbi.nlm.nih.gov/omim]

14. Koshland ME: The coming of age of the immunoglobulin J chain. Annu Rev Immunol 1985, 3:425-453.

15. Karagianni P, Wong J: HDAC3: taking the SMRT-N-CoRrect road to repression. Oncogene 2007, 26:5439-5449.

16. Baker SG, Kramer BS: Identifying genes that contribute most to good classification in microarrays. BMC Bioinformatics 2006, 7:407.

17. Troyanskaya O, Cantor M, Sherlock G, Brown P, Hastie T, Tibshirani $R$, Botstein $D$, Altman RB: Missing value estimation methods for DNA microarrays. Bioinformatics 2001, 17:520-525.

18. Sprott JC, Numerical Recipes Software: Numerical recipes: routine and examples in BASIC New York (USA): Cambridge University Press; 1998.

19. Tsai CA, Hsueh $\mathrm{H}$, Chen J]: Estimation of false discovery rates in multiple testing: application to gene microarray data. Biometrics 2003, 59: |07|-|08|.

20. Tusher VG, Tibshirani R, Chu G: Significance analysis of microarrays applied to the ionizing radiation response. Proc Natl Acad Sci USA 2001, 98:5116-5121.

21. Bamber D: The Area above the Ordinal Dominance Graph and the Area below the Receiver Operating Characteristic Graph. Journal of Math Psychology 1975, I 2:387-4I5.

Publish with Bio Med Central and every scientist can read your work free of charge

"BioMed Central will be the most significant development for disseminating the results of biomedical research in our lifetime. "

Sir Paul Nurse, Cancer Research UK

Your research papers will be:

- available free of charge to the entire biomedical community

- peer reviewed and published immediately upon acceptance

- cited in PubMed and archived on PubMed Central

- yours - you keep the copyright 